\title{
Planning Continuous Curvature Paths Using Constructive Polylines
}

Joshua H. Henrie

Brigham Young University - Provo

Follow this and additional works at: https://scholarsarchive.byu.edu/etd

Part of the Electrical and Computer Engineering Commons

\section{BYU ScholarsArchive Citation}

Henrie, Joshua H., "Planning Continuous Curvature Paths Using Constructive Polylines" (2008). Theses and Dissertations. 1514.

https://scholarsarchive.byu.edu/etd/1514

This Thesis is brought to you for free and open access by BYU ScholarsArchive. It has been accepted for inclusion in Theses and Dissertations by an authorized administrator of BYU ScholarsArchive. For more information, please contact scholarsarchive@byu.edu, ellen_amatangelo@byu.edu. 


\title{
PLANNING CONTINUOUS CURVATURE PATHS
}

\section{USING CONSTRUCTIVE POLYLINES}

by

\author{
Joshua Henrie
}

A thesis submitted to the faculty of

Brigham Young University

in partial fulfillment of the requirements for the degree of

Master of Science

Department of Electrical and Computer Engineering

Brigham Young University

August 2008 

Copyright (c) 2008 Joshua Henrie

All Rights Reserved 



\title{
BRIGHAM YOUNG UNIVERSITY
}

\section{GRADUATE COMMITTEE APPROVAL}

\author{
of a thesis submitted by
}

\author{
Joshua Henrie
}

This thesis has been read by each member of the following graduate committee and by majority vote has been found to be satisfactory.

Date

Date

Date
Doran K. Wilde, Chair

Clark N. Taylor

James K. Archibald 



\section{BRIGHAM YOUNG UNIVERSITY}

As chair of the candidate's graduate committee, I have read the thesis of Joshua Henrie in its final form and have found that (1) its format, citations, and bibliographical style are consistent and acceptable and fulfill university and department style requirements; (2) its illustrative materials including figures, tables, and charts are in place; and (3) the final manuscript is satisfactory to the graduate committee and is ready for submission to the university library.

Date

Accepted for the Department
Doran K. Wilde

Chair, Graduate Committee
Michael J. Wirthlin

Graduate Coordinator

Accepted for the College 



\author{
ABSTRACT \\ PLANNING CONTINUOUS CURVATURE PATHS \\ USING CONSTRUCTIVE POLYLINES \\ Joshua Henrie \\ Department of Electrical and Computer Engineering \\ Master of Science
}

Previous methods for planning clothoid based continuous curvature paths aim at minimizing path length. However, minimal length paths are not always smooth, natural, and drivable. A method of generating clothoid-based trajectories is discussed using constructive polylines. The goal of the motion planner is to create a path for a large car-like vehicle in human driving environments. Thus, the trajectories generated by the motion planner must be smooth, drivable, and natural such that the vehicle can follow the planned path on human roadways. Several examples are shown of trajectories developed for a DARPA Urban Challenge vehicle and a method of testing the motion planner and the vehicle controller is described. 



\section{ACKNOWLEDGMENTS}

Thank you to all who have supported, encouraged, and provided the opportunity to write this thesis. I especially thank:

My wife, Heather, I could not ask for a better companion.

Dr. Doran Wilde for the conversations and the insights that he provided.

My parents for instilling in me a desire for learning and education.

And my children for teaching me the joy and love that can be had in life. 



\section{Table of Contents}

Acknowledgments $\quad$ xi

List of Tables $\quad$ xvii

List of Figures $\quad$ xxi

$\begin{array}{ll}\text { Nomenclature } & \text { xxiii }\end{array}$

1 Introduction 1

1.1 Previous Work ........................ 1

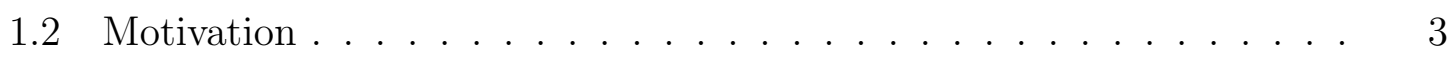

1.3 Problem Definition . . . . . . . . . . . . . . . . 4

1.3.1 Scaling Clothoids Using Constructive Polylines . . . . . . . . . 5

1.3.2 Smooth, Natural, and Drivable Trajectories . . . . . . . 5

1.3.3 Use of Waypoint Headings and Constructive Polyline Angles to Determine Maneuver Execution . . . . . . . . . . 5

1.3.4 Point Reduction Algorithm . . . . . . . . . . . . . 6

1.4 Overview of the Motion Planner . . . . . . . . . . . . . . 6

2 Clothoid Equations and Planning a Basic Clothoid Curve 9

2.1 Introduction . . . . . . . . . . . . . . . 9

2.2 Converting the Clothoid Equation . . . . . . . . . . . . 9

2.3 Translating Clothoid Equation to the $x y$ Plane . . . . . . . . . . 13 
2.4 Rotating and Scaling Clothoids . . . . . . . . . . . . . . 14

2.4.1 Rotating and Scaling Algorithm . . . . . . . . . . . . . 14

2.5 Conclusion . . . . . . . . . . . . . . . . 16

3 Constructing Trajectories Using Clothoids, Arcs, and Lines 17

3.1 Introduction . . . . . . . . . . . . . . . . 17

3.2 Constructing Trajectories . . . . . . . . . . . . . . . 18

3.2.1 Adding Arcs to Trajectories . . . . . . . . . . . . 18

3.2.2 Creating a Clothoid-Arc Set . . . . . . . . . . 21

3.3 Conclusion . . . . . . . . . . . . . . . . . . . . . . 27

4 Application of Trajectories $\quad 29$

4.1 Introduction . . . . . . . . . . . . . . . . 29

4.2 Generic Turn Trajectory . . . . . . . . . . . . . . . 29

4.2.1 Generic Turn Algorithm . . . . . . . . . . . . . . 30

4.3 Special Case Maneuvers . . . . . . . . . . . . . . . . . 38

4.3.1 Trajectories for Turns and Straight Segments . . . . . . . . 38

4.3.2 Lane Change Trajectory . . . . . . . . . . . . . . 40

4.3.3 U-turn Trajectory . . . . . . . . . . . . . . . 41

4.4 When to Execute a Trajectory Maneuver . . . . . . . . . . . . . 41

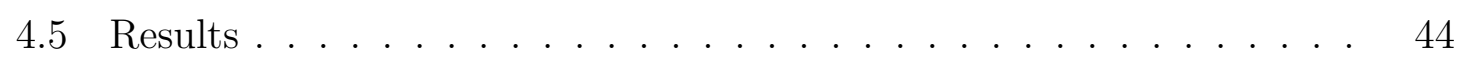

4.5.1 Motion Planning a Test Course . . . . . . . . . . . . 47

4.6 Conclusion . . . . . . . . . . . . . . . . . . . . 47

5 Testing the Motion Planner $\quad 51$

5.1 Introduction . . . . . . . . . . . . . 51

5.2 Obtaining Control and Test Data . . . . . . . . . . . . . 52 
5.2.1 Control Data . . . . . . . . . . . . . . . 52

5.2 .2 Testing Strategy _. . . . . . . . . . . . . . 52

5.3 Point Reduction Algorithm . . . . . . . . . . . . . . . . . 53

5.3.1 Finding the Curvature of a Point . . . . . . . . . . . 54

5.3.2 Douglas-Peucker Algorithm . . . . . . . . . . . . . . 56

5.3.3 Choosing a New Waypoint . . . . . . . . . . . . . . . . 57

5.4 Example Tests of the Motion Planner . . . . . . . . . . . . . . . . 58

5.4 .1 Test $1 \ldots \ldots \ldots \ldots \ldots$

5.4 Test $2 \ldots \ldots \ldots \ldots \ldots$

5.5 Conclusion . . . . . . . . . . . . . . . . 63

$\begin{array}{lll}6 & \text { Conclusion } & 65\end{array}$

6.1 Accomplishments . . . . . . . . . . . . . . . 66

6.2 Future Research . . . . . . . . . . . . . . . . . 66

$\begin{array}{ll}\text { Bibliography } & 69\end{array}$

$\begin{array}{lr}\text { File Listing } & 71\end{array}$ 


\section{List of Tables}

3.1 Restrictions placed when adding circular arcs to clothoids . . . . . . . 27

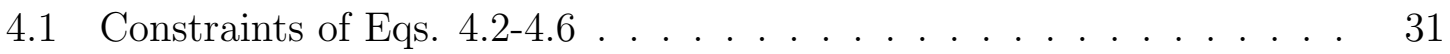

4.2 Generic turn with null space parameter $q$ added to constraints . . . . 32

4.3 Constraints for the solution $A=\delta_{0}$ and $B=\delta_{f} \ldots \ldots$. . . . . . 34

4.4 Constraints for the solution $A=\delta_{f}, B=\delta_{0}$, and free paramter $q \ldots 35$

4.5 Constraints for the straight-curve trajectory for $q=\frac{-\psi_{0}}{2}$ (see Table 4.4) 39

4.6 Constraints for the curve-straight trajectory for $q=\frac{\psi_{f}}{2}$ (see Table 4.4) 40

4.7 Lane change trajectory constraints for no $q$ and $\theta_{0}=\theta_{f}$ (see Table 4.4) 41

4.8 The distance, $d$, between the origin and each of the end waypoints, $x_{f}, y_{f}$, in Fig. 4.9. . . . . . . . . . . . . . . . . 45 
xviii 


\section{List of Figures}

1.1 (a) A trajectory (dash-dotted line) and the associated constructive polyline (green lines). (b) The curvature plot for the trajectory on the

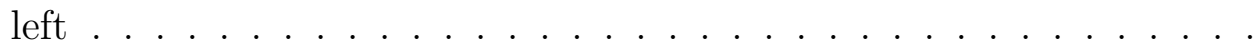

1.2 (a) Clothoid segment given heading a deflection $\delta$ and polyline length $d$. (b) Clothoid-arc segment given heading deflection $\delta$ and polyline length $d$. (c) Curvature diagram for clothoid segment with arc-length $L$, final curvature $\kappa$, sharpness (slope) $\alpha$, and deflection $\delta$ (area under the curve). (d) Curvature diagram for clothoid-arc segment with arclength $L$, final arc curvature $\kappa$, sharpness $\alpha$, and deflection $\delta$. . . .

1.3 (a) Symmetric curve. (b) Asymmetric curve. (c) Symmetric curvature diagram. (d) Asymmetric curvature diagram . . . . . . . . . . .

2.1 A clothoid in the $(a) s-\kappa$ domain and $(b) x y$ domain $\ldots \ldots \ldots \ldots$

2.2 Clothoid scaling. Clothoids in the $x y$ plane $(a)$ when $\kappa=1$ and $(b)$ scaled by $\frac{1}{|\kappa|} \ldots \ldots \ldots \ldots \ldots \ldots \ldots \ldots$

2.3 Clothoid_Turn() algorithm. Outputs of the algorithm are clothoid curvature, $\kappa$, sharpness, $\alpha$, and length, $L \ldots \ldots \ldots \ldots$

3.1 Symmetric clothoid set with unconstrained curvature in the $(a) s \kappa$ plane and $(b) x y$ plane . . . . . . . . . . . . . . .

3.2 Clothoid set with an inserted symmetric arc to constrain curvature in the $(a) s \kappa$ plane and (b) $x y$ plane. Note that $d_{1}$ is less than the $d$ in Eq. 3.1, and $d_{3}$ makes up the remaining distance to the halfway point between the initial and final points of the desired trajectory. $L_{c}$ is the length of the clothoid and $L_{\text {arc }}$ is the length of the arc. The angle of the arc, $\lambda$ (i.e., the change of heading of the arc), is a part of the change of heading of half the trajectory . . . . . . . . . . 
3.3 The black line shows a motion planned trajectory with curvature exceeding $\kappa_{\max }$. The blue lines shows the path a vehicle would make while trying to follow the black trajectory. The paths in the $(a) s \kappa$

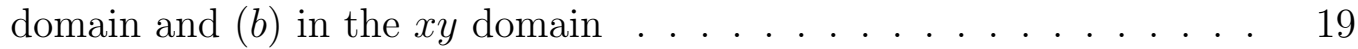

3.5 Clothoid set with an inserted asymmetric arc to constrain curvature . 20

3.6 Algorithm for finding half of the angle of an inserted arc, $\lambda$, using Newton's root approximation ............ . . 24

3.7 Example of the curvature of a trajectory when $\lambda>\delta \ldots . . . .25$

3.8 Clothoid_Turn() algorithm that includes arcs. Outputs of the algorithm are clothoid curvature, $\kappa$, sharpness, $\alpha$, length of the clothoid, $L$, and length of the arc, $L_{a r c} \ldots \ldots \ldots$

4.1 Generic turn trajectory in the $(a) s \kappa$ plane and (b) $x y$ plane. (Note that line $d$ here is different from line $d$ in Fig. 3.1b) . . . . . . . . 32

4.2 Algorithm for a symmetric generic turn trajectory. The output of the generic turn algorithm is a trajectory of four concatenated clothoid turns 37

4.3 (a) Undesirable shallow left-turn with a possible collision. (b) A leftturn consisting of a curve and a straight segment to avoid a collision . 39

4.4 (a) Straight-curve trajectory. (b) Curve-straight trajectory . . . . . . 39

4.5 Lane change maneuver . . . . . . . . . . . . . . . . . . . 41

4.6 Algorithm for a symmetric generic turn trajectory with added lane changes. The output of the algorithm is a trajectory of four concatenated clothoid turns . . . . . . . . . . . . . . 42

4.7 (a) U-turn trajectory. (b) U-turn trajectory with an arc . . . . . . 43

4.8 Using $\psi_{0}$ and $\delta$ to determine which maneuver to execute . . . . . 43

4.9 Chosen waypoints for left-turn trajectories. Each left-turn starts at the origin and ends at one of the waypoints. . . . . . . . . . 46

4.10 Maximum curvature versus length of $d$ for each of the left-turns in Fig. 4.9 for both the motion planner and Fraichard and Scheuer's method. 
4.11 (a) Example course with hand picked waypoints. (b) Results of the motion planner after the waypoints are passed to the motion planner. The motion planner uses each of the maneuvers discussed previously to plan the example course; straight, left-turn, right-turn, curve-straight, straight-curve, left and right lane changes, left u-turn, and the generic turn. The trajectories of the motion planner are plotted every 0.25 meters. . . . . . . . . . . . . . . . .

5.1 Logging control data with the vehicle system while manually driving the vehicle . . . . . . . . . . . . . . . . . . .

5.2 Finding the curvature of a waypoint from the headings and the distance between the two waypoints . . . . . . . . . . . . . . .

5.3 All four waypoints would be removed because they do not have zero or near zero curvature. The motion planner would not correctly generate the desired trajectories because of a missing waypoint at the inflection point. A waypoint must be added at the inflection point . . . . . . .

5.4 Driving the vehicle by reading a log file containing waypoints and using the Point Reduction Algorithm to choose the waypoints and passing them to the motion planner and vehicle controller . . . . . . . . . .

5.5 (a) A manually driven course with over 2400 waypoints. (b) The waypoints remaining after running the Point Reduction Algorithm on the manually driven course with a DP tolerance of 0.6 meters. These remaining waypoints are passed to the motion planner to create a list of trajectories to recreate the course . . . . . . . . . . . .

5.6 Comparison of the first test manual driven course and the course created by the motion planner from the PRA selected points . . . . . . .

5.7 (a) A manually driven course with nearly 1000 waypoints. (b) The waypoints remaining after running the Point Reduction Algorithm on the manually driven course with a DP tolerance of 0.4 meters. These remaining waypoints are passed to the motion planner to create a list of trajectories to recreate the course . . . . . . . . . . . .

5.8 (a) Comparison of the manual driven course and the course created by the motion planner with a DP tolerance of 0.4 meters and a nearzero curvature tolerance of $0.001 \frac{1}{m}$. (b) Comparison of the manual driven course and the course created by the motion planner with a DP tolerance of 0.6 meters and a near-zero curvature tolerance of $0.01 \frac{1}{m}$. 


\section{Nomenclature}

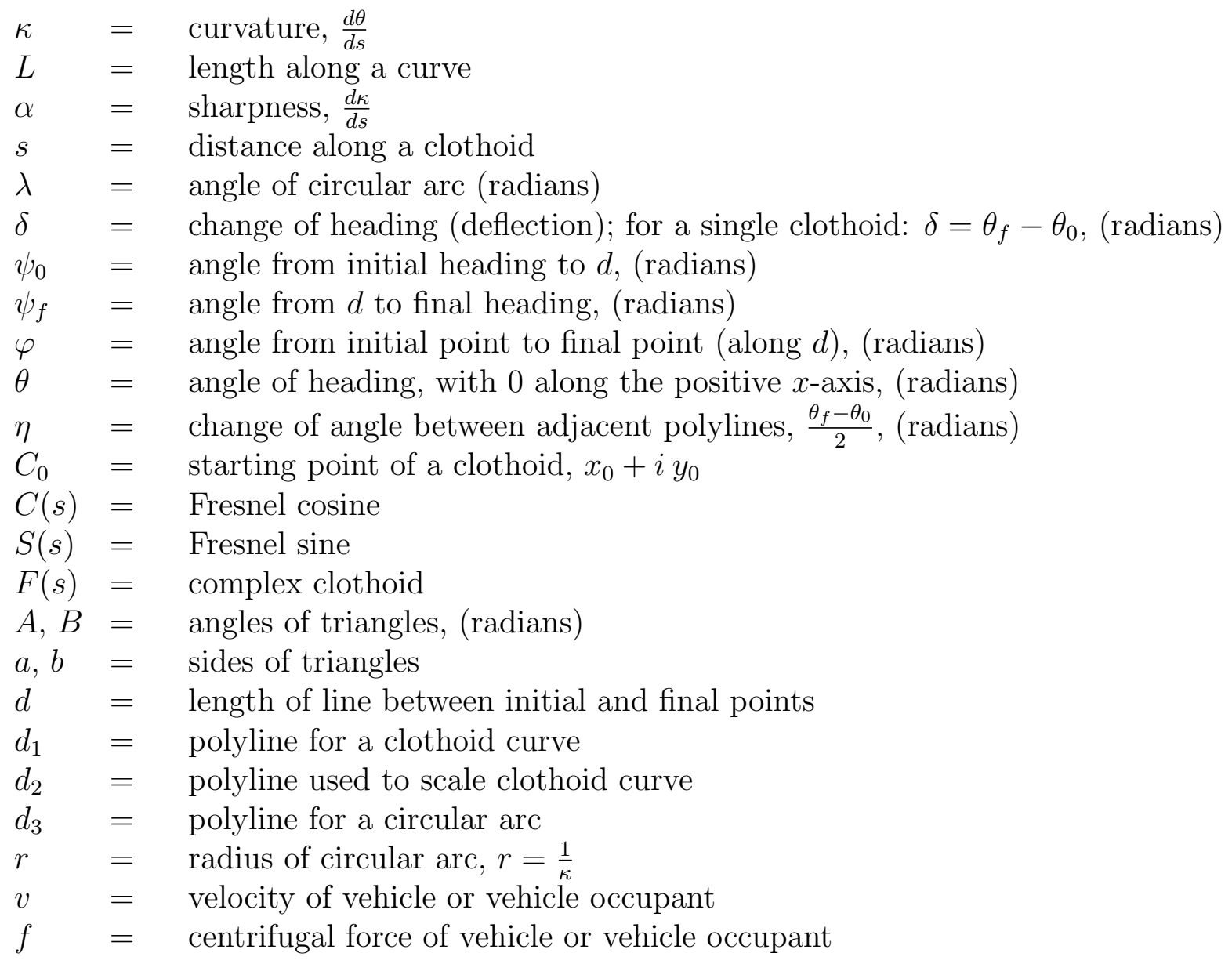

Subscripts

()$_{\max }=$ maximum

()$_{0}=$ initial

()$_{f}=$ final

()$_{c}=$ values pertaining to a clothoid 
xxiv 


\section{Chapter 1}

\section{Introduction}

The purpose of motion planning is to provide a path that autonomous car-like vehicles can physically drive. Motion planning has been defined as the study of open loop controls that steer a mobile robot from a specified initial state to a specified final state over a certain time interval [1]. Motion planning can be done by constructing trajectories that have steering controls (curvature and sharpness) as well as velocity controls (velocity and acceleration/deceleration) embedded in the data structure of the trajectory. The motion planner described in this thesis constructs smooth, natural, drivable trajectories for a large car-like vehicle. The planner generates these trajectories from constructive polylines using a combination of straight lines, clothoids, and circular arcs. A clothoid is a curve that increases/decreases linearly in curvature as it increases in arc length; curvature is the inverse of the radius and sharpness is the rate of change of curvature with respect to distance traveled. Constructive polylines are straight lines that are used to determine the desired curvature, sharpness, and length of a clothoid or the length of a circular arc of a trajectory.

\subsection{Previous Work}

Two commonly used clothoid motion planning methods are (1.) pre-compute the clothoids and heuristically choose the best clothoid path that arrives at the desired destination and heading, used by Coombs et al., Knowles, and Pivtoraiko and Kelly ([2], [3], [4]), and (2.) calculate a clothoid(s) that traverses the distance between two

points and ends with the desired heading. Both Shin and Singh, and Fraichard and Scheuer use this second method ([5], [6]). 
Pre-computing the clothoids and heuristically choosing the best path works well for motion planners integrated in with path planners. Where a path planner plans waypoints, points containing an $x y$ position and a heading, or paths that avoid objects and move the vehicle toward its desired destination and a motion planner plans a path between some given number of waypoints based on vehicle physical constraints such as turning radius. However, the vehicle system for which this motion planner was designed is a modular system. Therefore, it is assumed that a path planner has planned a route with waypoints and then passes the waypoints to the motion planner where trajectories are generated. A trajectory is a path that an autonomous vehicle follows to achieve the desired destination.

Unlike Shin and Singh, who use the curvature of postures (postures are described by a point and the heading and curvature at that point) to determine what types of clothoids to connect the postures [5], the motion planner described in this thesis uses line geometry between two points and their headings to generate trajectories. Fraichard and Scheuer also use geometry to calculate trajectories; however, they use the geometry of circles and lines to produce near optimal paths.

Paths called "optimal paths" in the previous literature refer to minimal length paths. Dubins showed that a length-optimal discontinuous curvature path connecting an initial state and a final state of a mobile robot consists of only straight line segments and circular arc segments of maximal allowable curvature [7]. Reeds and Shepp built upon Dubins' work and gave a complete characterization of the possible shortest discontinuous curvature paths [8]. They also extended Dubins' work by adding cusps (reversals in travel direction) to their paths, and enumerated the possible types of optimal paths showing that an optimal path required no more than five segments and no more than two direction reversals. Fraichard and Scheuer showed that under the continuous curvature assumption, near optimal paths consist of straight lines, circular arc segments of maximal allowable curvature, and clothoid curves [6]. These curves have maximum allowable sharpness and connect the straight-line segments to the circular arc segments. Remember that sharpness is change of curvature with respect to distance traveled and is also linearly related to the amount of steering motion 
along the trajectory [5] (i.e., related to the rate at which the steering wheel of the autonomous car-like vehicle turns). Fraichard and Scheuer paths are near optimal and within $10 \%$ of the length of Reeds and Shepp paths [6].

\subsection{Motivation}

Motivation for this motion planner is to generate a smooth, natural, drivable trajectory between two waypoints in forward motion similar to the way a human drives. Humans do not drive paths that have discontinuous curvature or only turn at maximum allowable curvature and sharpness like those generated by the prior work discussed above. For example, if a human were to drive paths that always made turns at maximum sharpness and curvature, the driving experience would be akin to that of driving with a teenager who tries to turn as sharply as possible at every turn. Such a driving experience would be uncomfortable and probably scary. The motion planner presented in this thesis uses the smallest curvature and sharpness required to obtain a trajectory between two waypoints. In other words the trajectory planned by the motion planner turns the steering wheel of a car-like vehicle as little as possible.

Once a path has been generated by the motion planner, the maximum safe velocity for the trajectory is calculated, and hence, the travel time is minimized along the trajectory. Since time-optimal trajectories do not necessarily require that car-like robots or vehicles perform turns at maximal sharpness and curvature, I have been investigating methods for constructing continuous curvature trajectories where the curvature and sharpness are left as variables. Therefore, instead of constructing paths/trajectories from maximal curvature circular arcs, maximal sharpness clothoids, and line segments, I investigate a new method for computing trajectories based on constructive polylines that allows curvature and sharpness of the trajectory to be computed as a function of the distance traveled and the desired change of heading.

Before proceeding further, the terms smooth, drivable, and natural need to be defined. Smooth means the curvature of the trajectory is continuous. Drivable means the trajectories are acceptable for driving on human roadways. For example, while 
left u-turns are acceptable on most roadways, right u-turns are not. The last term, natural, is more difficult to define, but essentially it means that motion planned paths are similar to paths that a human would drive under normal driving conditions (e.g., little or no traffic, no icy/rainy conditions, etc.). While driving in these conditions, humans do not continually turn the steering wheel as quickly and sharply as possible to make a turn. This behavior is unnatural for humans, as they tend to turn the steering wheel as little as possible, and when they do turn, they turn only as fast and sharply as is required to make the turn. While human behavior is difficult to quantize, the motion planner described in this thesis uses the smallest sharpness and curvature that is required to generate a path with the desired ending point and heading.

From these definitions of smooth, drivable, and natural, Dubins, and also Reeds and Shepp, paths are not smooth, drivable, or natural, as they do not have continuous curvature and require every turn to have maximum sharpness ([7], [8]). Fraichard and Scheuer paths are smooth and drivable, but not natural, as they require every turn to have maximum sharpness and curvature.

\subsection{Problem Definition}

The major contributions of this thesis to the community are the development and use of the following:

- constructive polylines to scale clothoids while retaining a clothoid's shape and deflection;

- straight lines, circular arcs, and scaled clothoids to create a family of smooth, natural, and drivable trajectories between given starting and ending waypoints;

- trajectory deflection and the angle between the initial heading and the constructive polyline to determine which maneuver to execute; and

- an algorithm to read waypoints from a file and select a few waypoints based on their position and headings to pass to the motion planner. 
The following sections briefly discuss each contribution. In addition to the contributions of this thesis is a publication in the Journal of Aerospace Computing, Information, and Communication written by the author and Wilde [9],

\subsubsection{Scaling Clothoids Using Constructive Polylines}

This thesis shows how to scale a clothoid by using a line (i.e., constructive polyline) that begins at the clothoid starting point and runs parallel to the clothoid ending point heading (Fig. 1.2a). This method of scaling clothoids was first published by the author and Wilde [9]. Using two or more constructive polylines to scale clothoids results in trajectories that an autonomous vehicle follows.

\subsubsection{Smooth, Natural, and Drivable Trajectories}

It is shown in this thesis how a family of trajectories are created that are smooth, natural, and drivable given starting and ending waypoints, and using a combination of straight lines, circular arcs, and scaled clothoids. Unlike previous works which require maximum sharpness and curvature, these trajectories have the minimal sharpness and curvature needed for a path to be created between the beginning and ending waypoints. This means that the vehicle turns only as quickly and sharply as required to end at the desired ending point and heading.

\subsubsection{Use of Waypoint Headings and Constructive Polyline Angles to Determine Maneuver Execution}

Because a family of trajectories exists, a method is needed to determine which member of the family of trajectories that the motion planner should generate. The method is developed in this thesis uses waypoint headings and constructive polyline angles to determine which maneuver (i.e., set of trajectories) to pass to the vehicle system. This method allows the motion planner to choose and create trajectories based on only two inputs, the length of the constructive polyline and the change of heading. 


\subsubsection{Point Reduction Algorithm}

A test course is used to test the motion planner and vehicle system. Because creating a test course by hand is a laborious and tedious process because points, distances, and headings must be chosen. Therefore, an automated process to generate a test course is desired. The Point Reduction Algorithm automates the test course process by reading in waypoints from a file containing thousands of waypoints which are recorded while manually driving the vehicle. The algorithm then selects a few waypoints based on their position, headings, and curvature. The selected waypoints are a test course and are passed to the motion planner. The motion planner is then tested by replicating the path of the recorded waypoints by generating trajectories between the selected waypoints. The generated trajectories can also be used to test the vehicle system by allowing the vehicle to drive the trajectories.

\subsection{Overview of the Motion Planner}

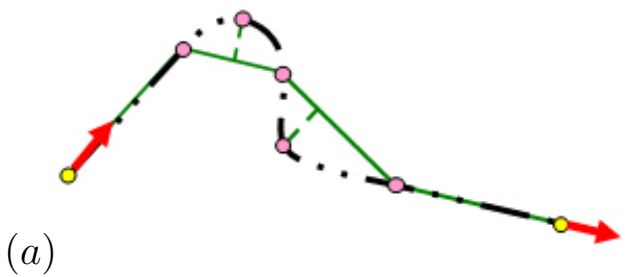

(a)

Figure 1.1: (a) A trajectory (dash-dotted line) and the associated constructive polyline (green lines). (b) The curvature plot for the trajectory on the left.

Clothoid-based trajectories are generated by the motion planner using constructive polylines. A constructive polyline connects the points where the curvature of the trajectory is zero, which includes straight lines and inflection points (Fig. 1.1). Furthermore, the polylines under curves are subdivided at the point where the clothoid or arc segment is parallel to the construction line. Figure 1.2 shows how a polyline is subdivided at the point of the curve when the polyline is parallel to the heading of the curve. These construction polylines are defined and used differently 
than the control lines used by Walton and Meek to draw clothoid arcs in a graphic editor $[10]$.

Chapter 2 describes an important primitive method, called Clothoid_Turn(), used for motion planning trajectories described by constructive polylines that has been proposed. The Clothoid_Turn() algorithm generates a curve by only using two values to calculate the arc length, $L$, the final curvature, $\kappa$, and the sharpness, $\alpha$ of the curve. The two values are: (1.) the length $d$ of the polyline segment, and (2.) the heading deflection, $\delta$, which is also the angle at which the curve is incident to the polyline.

Figure 1.2 shows two types of curves that can be generated by Clothoid_Turn(). Figures $1.2 \mathrm{a}$ and $1.2 \mathrm{~b}$ show the curves in the $x y$ domain, and Fig. $1.2 \mathrm{c}$ and $1.2 \mathrm{~d}$ show the curvature diagrams of Fig. $1.2 \mathrm{a}$ and $1.2 \mathrm{~b}$ respectively. A curvature diagram is convenient to work with because it visually relates the curvature (vertical axis), sharpness (slope), arc length (horizontal axis), and change of heading (area under the curve) of a clothoid.

Clothoid_Turn() generates a simple clothoid (Figs. 1.2a and 1.2c) if the final curvature is less than or equal to the maximum curvature the vehicle can drive, $\kappa_{\max }$. Otherwise, a combination clothoid-arc curve is generated (Figs. 1.2b and 1.2d) such that the curve does not exceed the $\kappa_{\max }$ of the vehicle. (Remember that $\kappa_{\max }$ is the inverse of the minimum turning radius of the vehicle.)

The Clothoid_Turn() method only really plans half a curve. If the curve is symmetric (Fig 1.3a and 1.3c), then a single call to the clothoid turn method plans both halves of the curve, and $d=\frac{\text { Length(polyline) }}{2}$. If the curve is asymmetric (Fig. $1.3 \mathrm{~b}$ and $1.3 \mathrm{~d}$ ), then two calls to the clothoid turn method must be made, where $d_{1}+d_{2}=$ Length(polyline). A curve of two clothoids that begins and ends with zero curvature is called a clothoid set.

Chapter 3 then describes how arcs are inserted into a clothoid set using Newton's root approximation method to find the angle and length of an arc. Chapter 4 uses the results of Chapters 2 and 3, in conjunction with polylines, to create trajectories from two points and their corresponding headings, similar to Fig. 1.1a. Finally, 


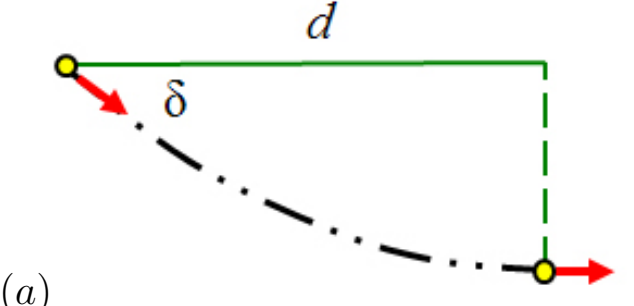

(a)

$(c)$

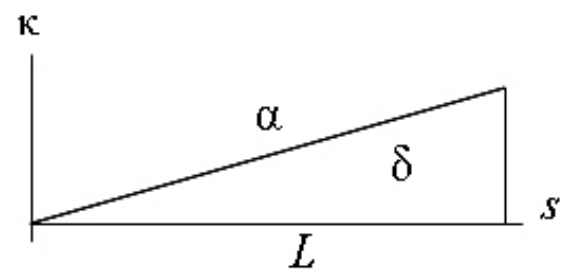

(b)

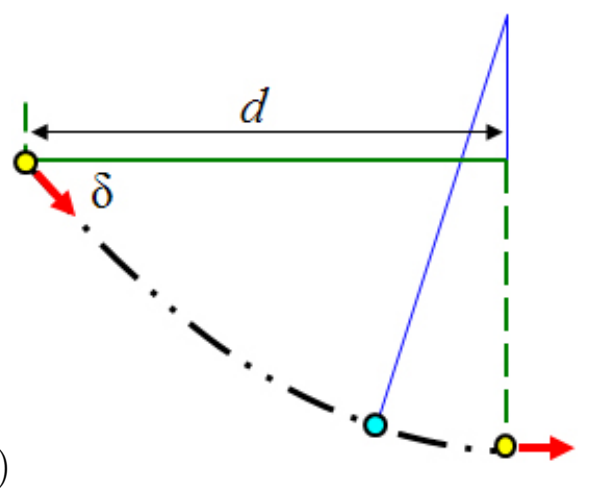

(d)

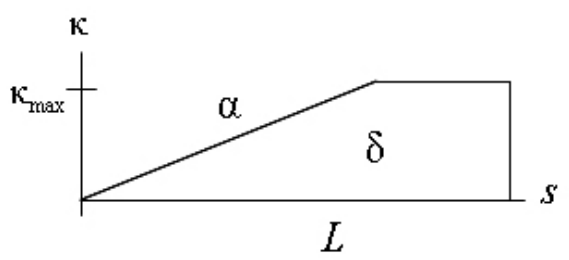

Figure 1.2: (a) Clothoid segment given heading a deflection $\delta$ and polyline length $d$. (b) Clothoid-arc segment given heading deflection $\delta$ and polyline length $d$. (c) Curvature diagram for clothoid segment with arc-length $L$, final curvature $\kappa$, sharpness (slope) $\alpha$, and deflection $\delta$ (area under the curve). ( $d$ ) Curvature diagram for clothoid-arc segment with arc-length $L$, final arc curvature $\kappa$, sharpness $\alpha$, and deflection $\delta$.

(a)

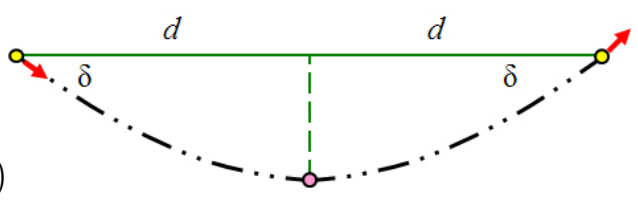

(c)

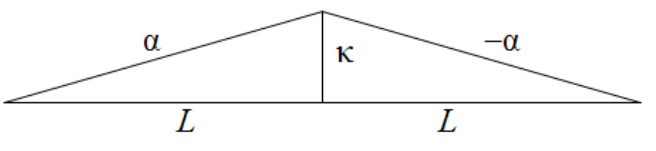

(b)

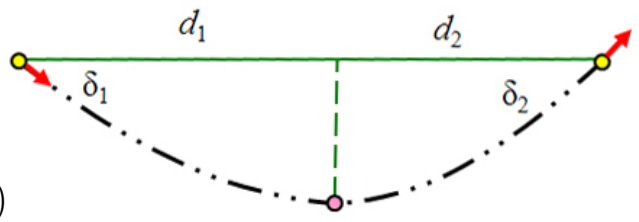

$(d)$

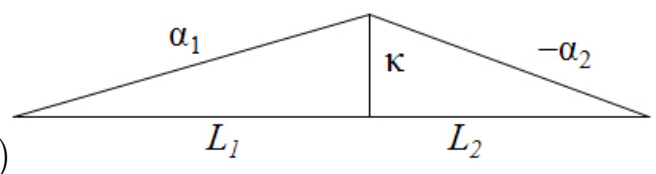

Figure 1.3: (a) Symmetric curve. (b) Asymmetric curve. (c) Symmetric curvature diagram. (d) Asymmetric curvature diagram.

in Chapter 5, the Point Reduction Algorithm is presented which creates a path description by reducing the number of points in a log file of GPS points and headings. The resulting waypoints are passed to the motion planner and the original path of the GPS points is replicated. The algorithm is used to test the motion planner and the vehicle system. 


\section{Chapter 2}

\section{Clothoid Equations and Planning a Basic Clothoid Curve}

\subsection{Introduction}

This chapter describes how a clothoid can be scaled without altering its shape and describes an algorithm which calculates a rotated and scaled clothoid with curvature, $\kappa$, sharpness, $\alpha$, and length, $L$, given a constructive polyline length, $d$, and change of heading, $\delta$.

First, a change of variables is performed on the clothoid equation, defined by Eq. 2.1, from two unknowns, clothoid sharpness, $\alpha$ and clothoid length, $s$, to one known and one unknown, clothoid change of heading, $\delta$, and clothoid curvature, $\kappa$, respectively. Then the clothoid equation is put into terms of the $x y$ plane because the constructive polyline, change of heading, and waypoints are in the $x y$ plane. Finally, the equation is scaled and rotated to create a trajectory of the correct length and orientation.

\subsection{Converting the Clothoid Equation}

A clothoid is a curve with continuous increasing/decreasing linear curvature and is defined in the complex plane by Eq. 2.1 derived by Kimia et al [11].

$$
\begin{aligned}
F(s)=C_{0} & +\operatorname{sign}(\alpha) \sqrt{\frac{\pi}{|\alpha|}} e^{i\left(\theta_{0}-\frac{\kappa_{0}^{2}}{2 \alpha}\right)}\left[C\left(\frac{\kappa_{0}+\alpha s}{\sqrt{\pi|\alpha|}}\right)-C\left(\frac{\kappa_{0}}{\sqrt{\pi|\alpha|}}\right)\right] \\
& +i \sqrt{\frac{\pi}{|\alpha|}} e^{i\left(\theta_{0}-\frac{\kappa_{0}^{2}}{2 \alpha}\right)}\left[S\left(\frac{\kappa_{0}+\alpha s}{\sqrt{\pi|\alpha|}}\right)-S\left(\frac{\kappa_{0}}{\sqrt{\pi|\alpha|}}\right)\right], \alpha \neq 0 .
\end{aligned}
$$


$C_{0}$ is the starting point of the clothoid; $\theta_{0}$ is the initial clothoid heading; $\kappa_{0}$ is the initial clothoid curvature; $\alpha$ is the clothoid sharpness; $s$ is the distance along the clothoid, and the Fresnel sine and cosine are defined as

$$
\begin{gathered}
C(s)=\int_{0}^{s} \cos \left(\frac{\pi}{2} u^{2}\right) d u \\
\text { and } S(s)=\int_{0}^{s} \sin \left(\frac{\pi}{2} u^{2}\right) d u \text {. }
\end{gathered}
$$

The term $\kappa_{0}+\alpha s$ is the instantaneous curvature after traveling a distance $s$ along the trajectory.

While Eq. 2.1 is valid for all clothoids except where $\alpha=0$, which is the case for straight lines and circular arcs, it is in terms of two unknown variables $\alpha$ and $s$. To reduce the number of unknowns, the clothoid equation is converted from the two unknown variables to one unknown variable, $\kappa$, and one known variable, $\delta$. The variable $\kappa$ was chosen to be the unknown variable in the equation, and not $\alpha$ or $s$, because of the clothoid scaling process, which is described in Section 2.4.

Converting Eq. 2.1 can be simplified by assuming that the initial and final points of a clothoid set begin and end with zero curvature, $\kappa_{0}=0$ (Fig. $1.3 \mathrm{c}$ and 1.3d). In essence, this means that the vehicle's wheels must be straight upon entering or exiting a trajectory. Assuming $\kappa_{0}=0$ greatly simplifies the process of creating continuous curvature trajectories by allowing the catenation of arbitrary clothoid sets and straight-line segments. If the vehicle exits a trajectory with non-zero curvature, an additional clothoid must be added to create a continuous curvature trajectory that can connect to a straight line. However, if the vehicle exits a trajectory with zero curvature then it may enter a tangential straight-line segment without needing to add additional clothoids for the transition.

$$
F(s)=\sqrt{\frac{\pi}{|\alpha|}}\left[\operatorname{sign}(\alpha) C\left(\frac{\alpha s}{\sqrt{\pi|\alpha|}}\right)+i S\left(\frac{\alpha s}{\sqrt{\pi|\alpha|}}\right)\right], \alpha \neq 0
$$



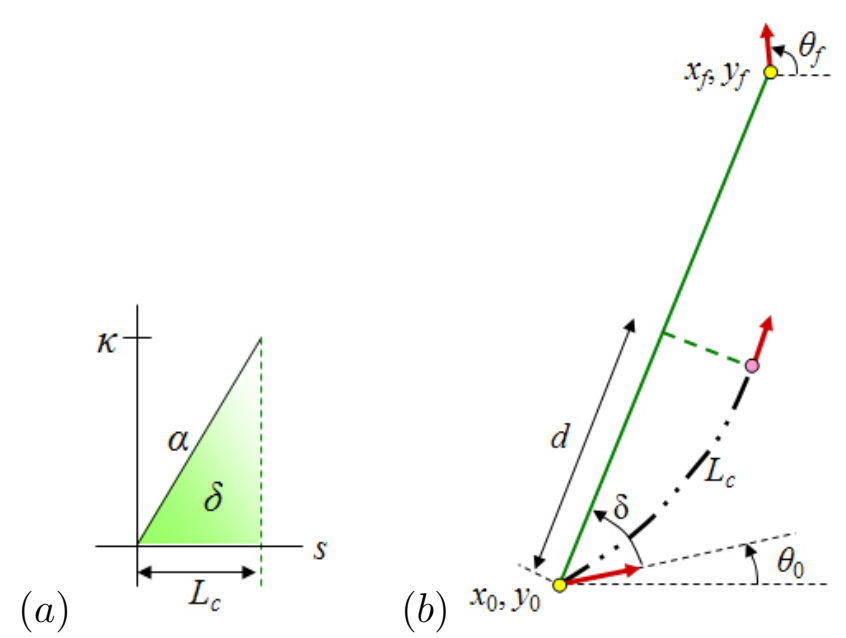

Figure 2.1: A clothoid in the $(a) s-\kappa$ domain and $(b) x y$ domain.

Without loss of generality, a trajectory can be computed for the normal case $\theta_{0}=0$ and $C_{0}=0$, and then rotated and translated into its correct orientation. Section 2.4.1 further explains the process of rotating and translating a clothoid curve. After applying the assumptions of $\kappa_{0}=0, \theta_{0}=0$ and $C_{0}=0$, Eq. 2.1 reduces to Eq. 2.4 .

Although Eq. 2.4 has been significantly reduced from Eq. 2.1, it is stated only in terms of the unknown curve length, $s$, and sharpness, $\alpha$. The inputs of the motion planner consist of only the initial and final points and their associated headings, $\theta_{0}$ and $\theta_{f}$. Therefore, the length, curvature, and sharpness of the desired clothoid for the trajectory are not inputs. The change in heading $\left(\delta=\theta_{f}-\theta_{0}\right)$, also known as deflection [6], is easily computed from the initial and final points. To derive the primitive clothoid turn method referred to in the introduction, Eq. 2.4 can be rewritten in terms of $\delta$ and $\kappa$, Eq. 2.9, instead of $s$ and $\alpha$. The purpose of this change of variables is to use $\delta$ and a contructive polyline to scale a clothoid linearly with $\kappa$. The process of determining the value of $\kappa$ and scaling a clothoid is discussed in Section 2.4. The derivation for Eq. 2.9 is given below.

As stated previously, a curvature diagram showing the curvature, $\kappa$, with respect to the distance traveled along the curve, $s$, (Fig. 2.1a) is a convenient diagram to work with because it visually relates the curvature (vertical axis), sharpness (slope), 
length (horizontal axis), and change of heading (area under the curve) of a clothoid. Figure 2.1 shows a clothoid in the $s-\kappa$ domain and the $x y$ domain. From this figure, the relationships between variables $\delta$ and $\kappa$ and variables $s$ and $\alpha$ are visible (Eqs. $2.5)$.

$$
\kappa=\alpha s, \delta=\frac{\alpha s^{2}}{2}, \alpha=\frac{2 \delta}{\kappa^{2}}
$$

Using the Eqs. 2.5, the inner terms of the Fresnel integrals in Eq. 2.4 are put into terms of $\delta$.

$$
\begin{aligned}
\frac{\alpha s}{\sqrt{\pi|\alpha|}}= & \left\{\begin{array}{l}
\alpha>0: \sqrt{\frac{\alpha^{2} s^{2}}{\pi \alpha}}=\sqrt{\frac{\alpha s^{2}}{\pi}}=\sqrt{\frac{2 \delta}{\pi}} \\
\alpha<0:(-1) \sqrt{\frac{(-\alpha)^{2} s^{2}}{\pi(-\alpha)}}=(-1) \sqrt{\frac{(-\alpha) s^{2}}{\pi}}=(-1) \sqrt{\frac{2(-\delta)}{\pi}}
\end{array}\right\} \\
= & \frac{\delta}{|\delta|} \sqrt{\frac{2|\delta|}{\pi}}=\operatorname{sign}(\delta) \sqrt{\frac{2|\delta|}{\pi}}
\end{aligned}
$$

where

$$
\operatorname{sign}(\alpha)=\frac{\alpha}{|\alpha|}=\frac{\kappa}{|\kappa|}=\frac{\delta}{|\delta|}=\operatorname{sign}(\delta) .
$$

For a single clothoid starting with zero curvature, Eq. 2.7 can be derived from Fig. 2.1.

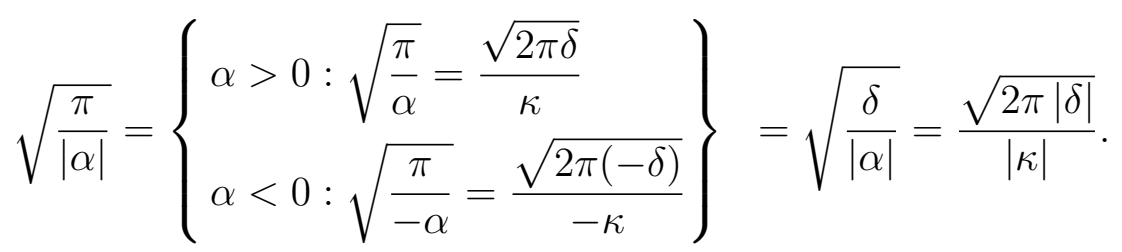

Next, a change of variables is performed on the constant term in front of the Fresnel equations in Eq. 2.4 to terms of $\delta$ and $\kappa$ by using the same Eqs. 2.5. Substituting Eqs. 2.6-2.7 into Eq. 2.4, the final transformed clothoid equation is

$$
F\left(\frac{2 \delta}{\kappa}\right)=\frac{\sqrt{2 \pi|\delta|}}{|\kappa|}\left[C\left(\sqrt{\frac{2|\delta|}{\pi}}\right)+\operatorname{sign}(\delta) i S\left(\sqrt{\frac{2|\delta|}{\pi}}\right)\right]
$$




\subsection{Translating Clothoid Equation to the $x y$ Plane}

As the constructive polylines, the change of heading, and the waypoints are in the $x y$ plane and the purpose of the constructive polylines is to scale clothoids in the $x y$ plane, it is advantageous to translate Eq. 2.9 into terms of the $x y$ plane. Translating the equation to the $x y$ plane also is the foundation for the clothoid scaling and rotating process discussed in Section 2.4. To translate let

$$
F\left(\frac{2 \delta}{\kappa}\right)=x\left(\frac{2 \delta}{\kappa}\right)+i y\left(\frac{2 \delta}{\kappa}\right)
$$

where

$$
\begin{gathered}
x\left(\frac{2 \delta}{\kappa}\right)=\frac{\sqrt{2 \pi|\delta|}}{|\kappa|} C\left(\sqrt{\frac{2|\delta|}{\pi}}\right) \\
\text { and } y\left(\frac{2 \delta}{\kappa}\right)=\operatorname{sign}(\delta) \frac{\sqrt{2 \pi|\delta|}}{|\kappa|} S\left(\sqrt{\frac{2|\delta|}{\pi}}\right) .
\end{gathered}
$$

An important and key observation from Eqs. 2.11 and 2.12 is that $\kappa$ determines the size of the clothoid and $\delta$ determines the shape of a clothoid. Therefore, $\kappa$ can be used to scale the size of a clothoid without altering its shape.

$$
\begin{gathered}
x\left(\frac{2 \delta}{\kappa}\right)=\frac{1}{|\kappa|} x(2 \delta) \\
\text { and } y\left(\frac{2 \delta}{\kappa}\right)=\frac{1}{|\kappa|} y(2 \delta)
\end{gathered}
$$

where the shape of the clothoid is determined by $\delta$ alone:

$$
\begin{gathered}
x(2 \delta)=\sqrt{2 \pi|\delta|} C\left(\sqrt{\frac{2|\delta|}{\pi}}\right) \\
\text { and } y(2 \delta)=\operatorname{sign}(\delta) \sqrt{2 \pi|\delta|} S\left(\sqrt{\frac{2|\delta|}{\pi}}\right) .
\end{gathered}
$$



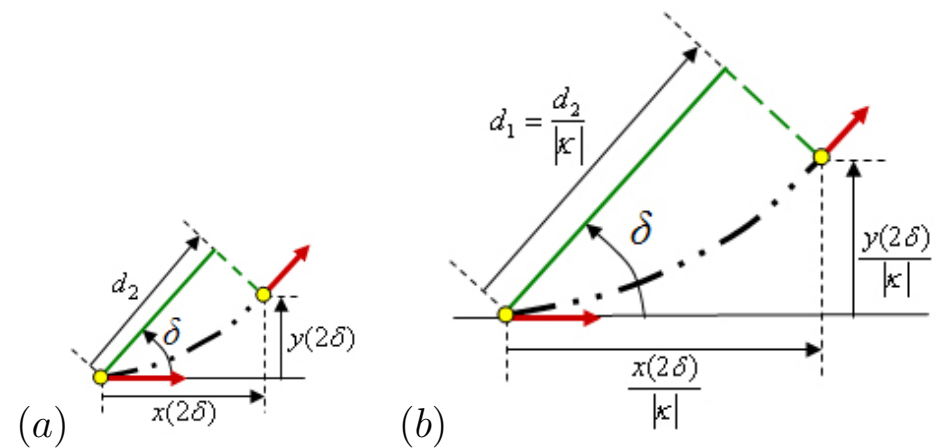

Figure 2.2: Clothoid scaling. Clothoids in the $x y$ plane $(a)$ when $\kappa=1$ and $(b)$ scaled by $\frac{1}{|\kappa|}$.

\subsection{Rotating and Scaling Clothoids}

The rotating and scaling of clothoids is a key result of this thesis and the basis of the proposed method to construct trajectories. The clothoid is rotated by $\delta$ and is scaled by first finding the shape of the clothoid and then calculating the desired curvature given the length of a constructive polyline.

For example, Fig. 2.2 shows similar clothoids where Fig. 2.2a is the desired shape of the clothoid and is produced from Eqs. 2.15 and 2.16 and Fig 2.2b is the desired scaled size of the clothoid and is produced from Eqs. 2.11 and 2.12. Remember that Eqs. 2.13 and 2.14 describe the relationship between the two figures.

Because the clothoids in Fig. 2.2 are similar, they share the same initial and final headings and deflection. However, $x, y, d_{2}$, and the length of the curve are all scaled linearly with $\frac{1}{|\kappa|}$. Thus, the distance between the beginning and ending points in Fig. 2.2b can be spanned by scaling the clothoid in Fig. 2.2a.

\subsubsection{Rotating and Scaling Algorithm}

To scale and rotate a clothoid first let $\kappa=1$ and compute $x(2 \delta)$ and $y(2 \delta)$, which are functions of only $\delta$. Next, rotate $x(2 \delta)$ and $y(2 \delta)$ by $-\delta$; the $x$-component of the resulting point is $d_{2}$ in Eq. 2.17.

$$
d_{2}=\cos (\delta) x(2 \delta)+\sin (\delta) y(2 \delta)
$$




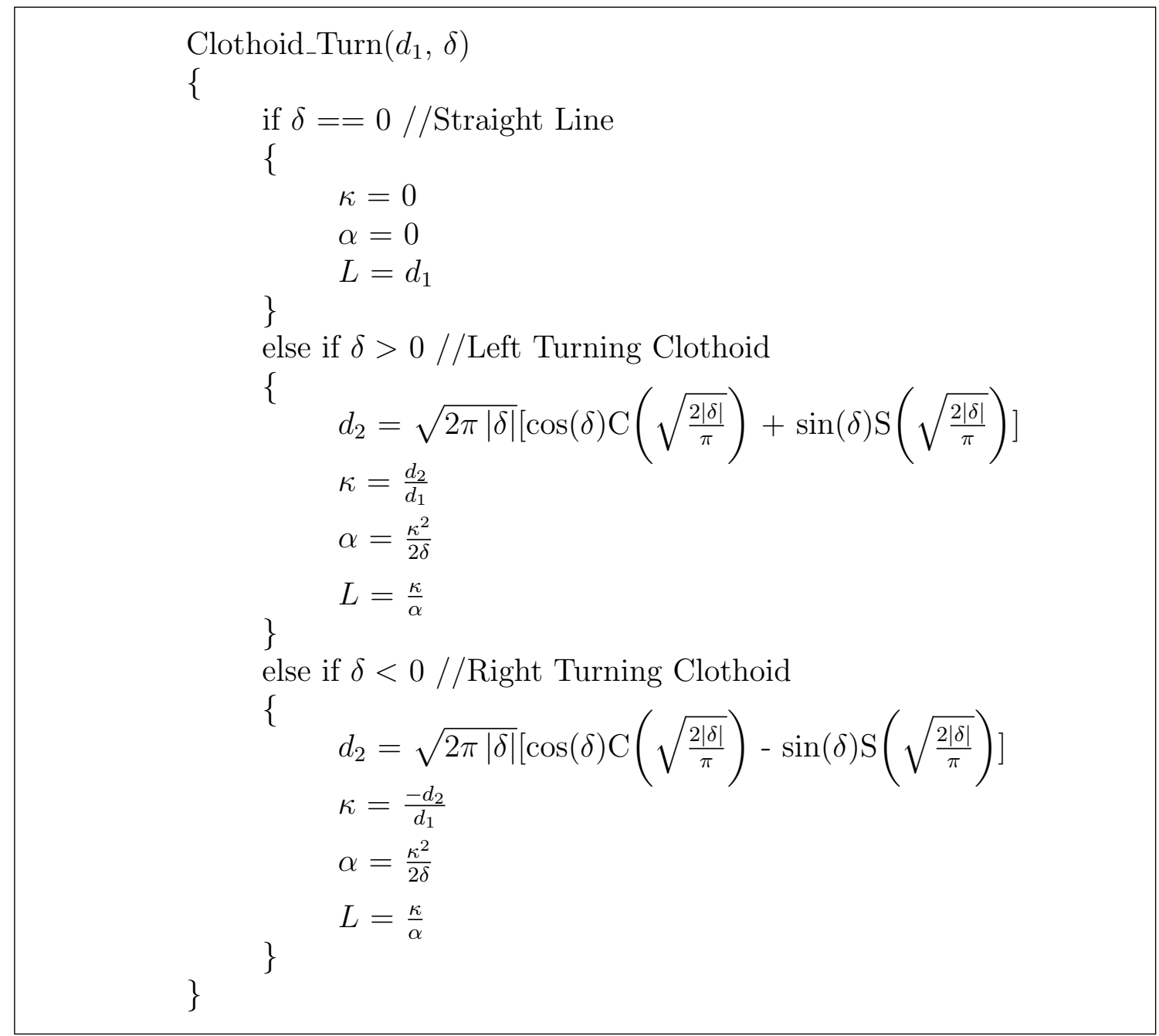

Figure 2.3: Clothoid_Turn() algorithm. Outputs of the algorithm are clothoid curvature, $\kappa$, sharpness, $\alpha$, and length, $L$.

As described in the next section, the distance $d_{1}$ in Fig. $2.2 \mathrm{~b}$ is computed by Eq. 3.1. After computing both $d_{1}$ and $d_{2}$ the curvature, $\kappa$, can be computed from Eq. 2.18.

$$
\begin{gathered}
\kappa=\operatorname{sign}(\delta) \frac{d_{2}}{d_{1}} . \\
\alpha=\frac{\kappa^{2}}{2 \delta}, L=\frac{\kappa}{\alpha} .
\end{gathered}
$$


Once $\kappa$ is computed, the length and sharpness of the scaled clothoid (Fig. 2.2b) can be found using Eq. 2.19. The result is a clothoid turn with the desired length and change of heading. The clothoid turn algorithm with inputs $d_{1}$ and $\delta$ and outputs $\kappa, \alpha$ and $L$ can be seen in Fig. 2.3.

\subsection{Conclusion}

The Clothoid_Turn() algorithm uses the property of clothoids that states that they can be scaled while retaining their shape and deflection to produce clothoids based on the length of a polyline and the desired change of heading. Using the algorithm, basic trajectories can be created from two points and their associated headings. 


\section{Chapter 3}

\section{Constructing Trajectories Using Clothoids, Arcs, and Lines}

\subsection{Introduction}

Trajectories can be planned from two points and their headings using polylines and the clothoid scaling process described by the Clothoid_Turn() algorithm. Because the motion planner is used for large car-like vehicles which have a large minimum turning radius (turning radius is the inverse of curvature), arcs must be used in the trajectories to upper-bound the curvature $\left(\max\right.$ curvature $\left.=\frac{1}{\text { min.radius }}\right)$. Therefore, trajectories for large car-like vehicles consist of circular arcs, straight lines, and clothoids. The clothoids are used to make smooth transitions between the straight lines, circular arcs, and other trajectories.

$(a)$
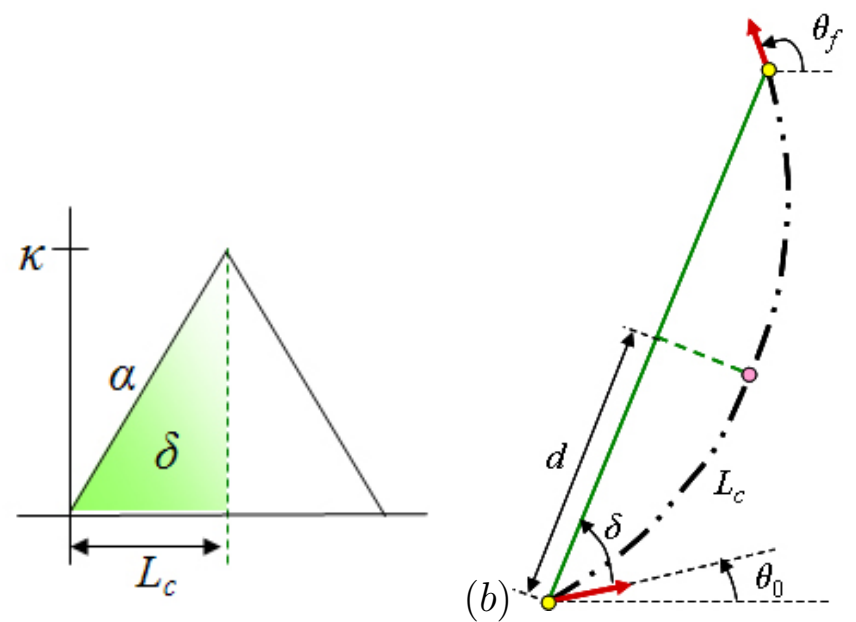

Figure 3.1: Symmetric clothoid set with unconstrained curvature in the $(a) s \kappa$ plane and $(b) x y$ plane. 


\subsection{Constructing Trajectories}

Remember that trajectories begin and end with zero curvature. Therefore, the simplest trajectory consists of a single straight line. Following the straight line, the next simplest trajectory consists of two clothoids of the same sharpness, the second clothoid mirroring the first (Fig. 3.1). Two clothoids with the same magnitude of sharpness but opposite signs are called a symmetric clothoid set.

If the change of heading of the clothoid set is positive then the trajectory is a left turn, and if the change of heading of the set is negative then the trajectory is a right turn. To create a symmetric clothoid set choose a constructive polyline with length $d$, where

$$
d=\frac{\sqrt{\left(x_{f}-x_{0}\right)^{2}+\left(y_{f}-y_{0}\right)^{2}}}{2} .
$$

A constructive polyline with length $d$ creates a clothoid turn that covers half the distance between the initial and final points. Mirroring the clothoid covers the second half of the distance and a trajectory is created (Fig. 3.1). It should be noted that choosing $d$ to be half the distance between the initial and final points is not required, but it is a simple and effective solution.

A clothoid set is a simple trajectory because it is based on a single construction polyline and begins and ends with zero curvature. At this point, the definition of a clothoid set is expanded to include an optional arc which is an upper-bound on curvature. Adding an arc to a clothoid set creates the same type of trajectory as in Fig. 3.1, but with upper-bounded curvature (Fig. 3.2). This extended definition of a clothoid set is similar to a "CC-Turn" by Fraichard and Scheuer [6], except it is not required to have maximum sharpness and curvature.

\subsubsection{Adding Arcs to Trajectories}

All vehicles have a minimum turning radius. Thus, arcs need to be introduced into the trajectories to ensure that the curvature of the trajectories does not exceed the vehicle's maximum curvature (i.e., minimum turning radius). Figure 3.3a shows 


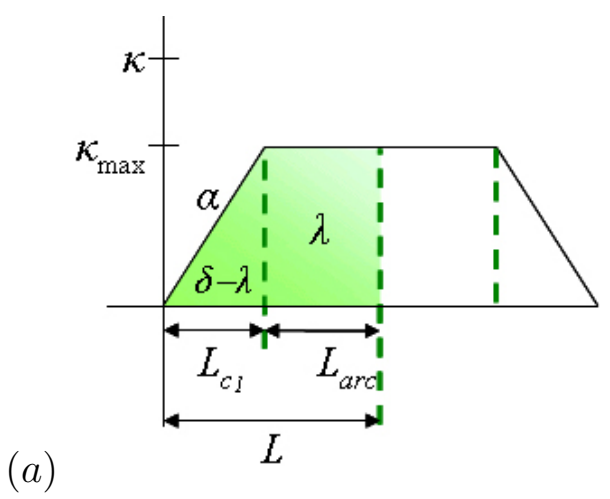

(b)

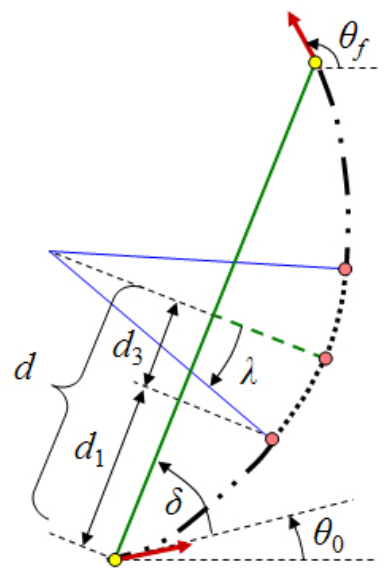

Figure 3.2: Clothoid set with an inserted symmetric arc to constrain curvature in the (a) $s \kappa$ plane and (b) $x y$ plane. Note that $d_{1}$ is less than the $d$ in Eq. 3.1, and $d_{3}$ makes up the remaining distance to the halfway point between the initial and final points of the desired trajectory. $L_{c}$ is the length of the clothoid and $L_{\text {arc }}$ is the length of the arc. The angle of the arc, $\lambda$ (i.e., the change of heading of the arc), is a part of the change of heading of half the trajectory.

an example of when a motion planned trajectory (black line) exceeds the maximum curvature of a vehicle, $\kappa_{\max }$. Figure $3.3 \mathrm{~b}$ shows an example of the vehicle (blue dashed line) trying to follow the planned trajectory (black dash-dot-dot line) but is unable to match the curvature of the trajectory because the curvature of the trajectory exceeds the maximum curvature the vehicle can achieve. The result is that the vehicle does not end at the desired ending waypoint.

(a)

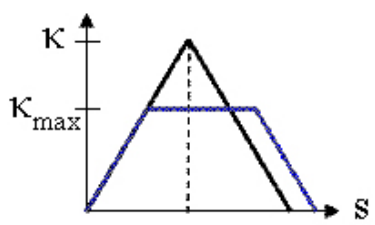

(b)

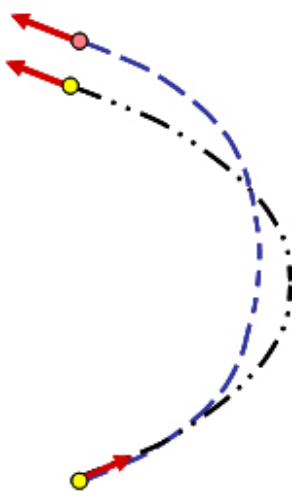

Figure 3.3: The black lines shows a motion planned trajectory with curvature exceeding $\kappa_{\max }$. The blue lines shows the path a vehicle would make while trying to follow the black trajectory. The paths in the $(a) s \kappa$ domain and $(b)$ in the $x y$ domain. 
However, by inserting an arc with $\kappa_{a r c}=\kappa_{\max }$ into the motion planned trajectory between the two clothoids, then the vehicle is able to follow the path because the new trajectory does not exceed $\kappa_{\max }$. Thus, the vehicle follows the path and ends at the desired ending waypoint.

(a)

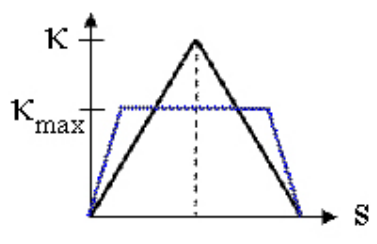

(b)

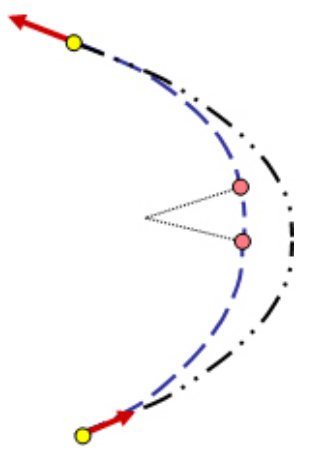

Figure 3.4: The black line shows a motion planned trajectory with curvature exceeding $\kappa_{\max }$. The blue line shows a motion planned trajectory that the vehicle could follow with $\kappa=\kappa_{\max }$. The paths in the $(a) s \kappa$ domain and $(b)$ in the $x y$ domain.

(a)

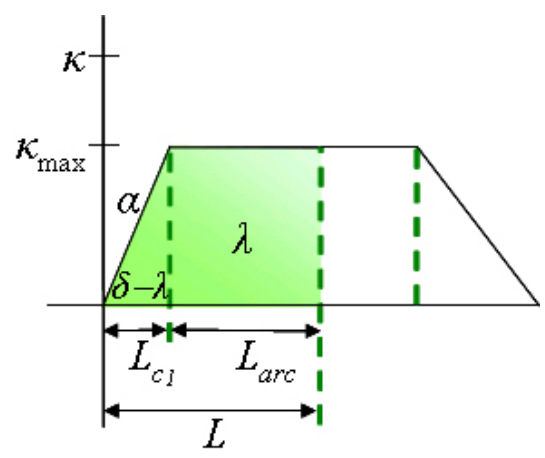

(b)

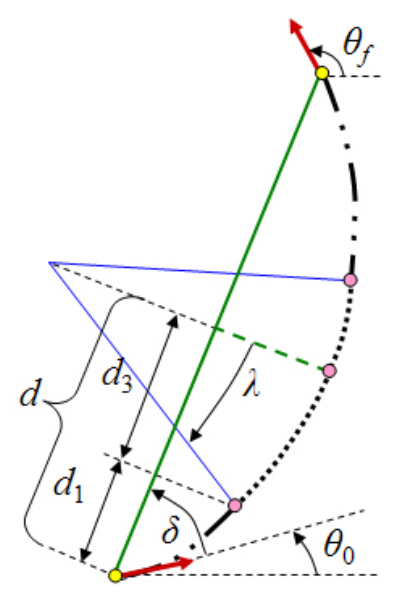

Figure 3.5: A trajectory with two clothoids of different sharpness and an arc to constrain curvature in $(a)$ the $s \kappa$ plane and $(b) x y$ plane. 


\subsubsection{Creating a Clothoid-Arc Set}

To create a clothoid set with an arc is similar process to that of creating a clothoid set without an arc. To develop a symmetric clothoid-arc set, first create a clothoid with a maximum curvature of $\kappa_{\max }$. Then add an arc to finish the remaining half of the distance between the beginning and ending points. The arc is then mirrored and a mirror of the first clothoid is added on the end (Fig. 3.2). The same principles of trajectory generation apply to asymmetric clothoid sets (Fig. 3.5).

Figure 3.1a shows a curvature vs. distance plot for a two clothoid trajectory with unrestrained curvature. If the maximum curvature of the two clothoids is greater than the maximum curvature, $\kappa_{\max }$, the vehicle is able to drive, the vehicle would be unable to follow the trajectory. Figure 3.2 shows that inserting an arc between the two clothoids with a radius, $r \geq \frac{1}{\kappa_{\max }}$, results in a trajectory that the vehicle can follow.

If the curvature of the planned clothoid set exceeds $k_{\max }$ then an arc is inserted between the beginning and ending clothoids. In order to insert an arc, the angle of the arc, $\lambda$, length of the arc, $L_{a r c}$, and the endpoint of the first clothoid must be determined. In the derivation following I find a method of computing $\lambda$.

Fortunately, it is advantageous to start with the change of heading, length, and curvature of the clothoid set already planned. Because the desired change in heading has not changed, the area $\delta$ in Fig. 3.1a must be equal to the area of half the trajectory in Fig. 3.2a. In other words, the area for $\delta$ in Fig. 3.1a is

$$
\delta=\frac{\kappa L_{c}}{2}
$$

and the area for half of the trajectory, i.e one clothoid and half the arc, in Fig. 3.2a is

$$
\delta=\frac{\kappa_{\max } L_{c_{1}}}{2}+\lambda
$$

where $\lambda$, the angle of the circular arc, is

$$
\lambda=\kappa_{\max } L_{\text {arc }} .
$$


Next, set Eq. 3.2 equal to Eq. 3.3.

$$
\frac{\kappa L_{c}}{2}=\frac{\kappa_{\max } L_{c_{1}}}{2}+\kappa_{\max } L_{\text {arc }}
$$

Also from Fig. 3.2a it can be seen that

$$
L=L_{c_{1}}+L_{a r c}
$$

Using Eq. 3.6 multiply both sides of the equation by $\kappa_{\max }$, and subtract the result from Eq. 3.5 and solve for $L_{c_{1}}$.

$$
L_{c_{1}}=2 L-L_{c} \frac{\kappa}{\kappa_{\max }} .
$$

Next, solve for $L_{c_{1}}$ in Eq. 3.3 and set the result equal to Eq. 3.7.

$$
\frac{2(\delta-\lambda)}{\kappa_{\max }}=2 L-L_{c} \frac{\kappa}{\kappa_{\max }} .
$$

Finally, solve for $\lambda$ in Eq. 3.8.

$$
\lambda=2 \delta-\kappa_{\max } L
$$

In the results above, $\lambda$ is half the angle of the total circular arc that is to be inserted into the trajectory. However, $\lambda, L, L_{1}$, and $L_{3}$ are all unknown and are dependent upon each other, thus, an approximation must be made to calculate $\lambda$. In the following section, a function $G(\lambda)$ is derived whose root is the correct $\lambda$. As Clothoid_Turn() returns $\kappa, L_{c}$, and $\alpha$, let $L=L_{c}$ to find an approximation for $\lambda$. Then by using Newton's root approximation method, similar to Walton and Meek [10], the correct values for $\lambda$ and $L$ can be determined. Once $\lambda$ and $L$ have been found, a trajectory can be created for a clothoid with length $L_{c_{1}}$, an arc with length $2 L_{\text {arc }}$ and angle $2 \lambda$, and finishing with a mirrored clothoid (Fig. 3.2). 


\section{Newton's Root Approximation}

Newton's root approximation method uses an iterative approach to successively calculate better approximations of zeros of a real function. Starting with an initial guess that is somewhat near the zero, the function is approximated by its tangent line. The value at which the tangent line crosses the $\mathrm{x}$-axis the becomes the new guess in the next iteration. The process repeats until the approximate zero is close enough to the true root.

To use Newton's root approximation method to find a good approximation for $\lambda$, a function, $G$, must be in terms of $\lambda$ and must be equal to zero when the root, $\lambda$, is found. To determine $G$, it can be seen from Fig. 3.2b that $d=d_{1}+d_{3}$, where $d$ is half the distance between the initial and final points, $d_{1}$ is the length of the projection of the clothoid onto $d$, and $d_{3}$ is the length of the projection of the arc onto the same line. Note that the length $d$ is the same as Eq. 3.1 and that the change of heading of a clothoid, $\delta$, is now $\delta-\lambda$.

From Eqs. 2.11 and 2.12, substituting the clothoid change of heading, $\delta$, for $\delta-\lambda$, and projecting $d_{1}$ onto $d$, it can be seen that $d_{1}$ is

$$
\begin{aligned}
& d_{1}=\cos (\delta) x\left(\frac{2(\delta-\lambda)}{\kappa}\right)+\sin (\delta) y\left(\frac{2(\delta-\lambda)}{\kappa}\right) \\
& =\frac{\sqrt{2 \pi|\delta-\lambda|}}{|\kappa|}\left[\cos (\delta) C\left(\sqrt{\frac{2|\delta-\lambda|}{\pi}}\right)+\operatorname{sign}(\delta-\lambda) \sin (\delta) S\left(\sqrt{\frac{2|\delta-\lambda|}{\pi}}\right)\right] .
\end{aligned}
$$

Using geometry in Fig. $3.2 \mathrm{~b}, d_{3}$ is found to be

$$
d_{3}=\frac{1}{|\kappa|}[\cos (\delta) \sin (\delta-\lambda)-\sin (\delta) \cos (\delta-\lambda)]
$$

Subtracting $d_{1}$ and $d_{3}$ from $d$ results in a function, $G$, that is zero when $\lambda$ is a root and nonzero when $\lambda$ is not a root.

$$
G=d-d_{1}-d_{3}
$$


Next, take the derivative of $G$ after substituting the equations for $d_{1}$ and $d_{3}$ in $G$, results in $G^{\prime}$.

$$
\begin{array}{r}
G^{\prime}=\frac{\pi \operatorname{sign}(\delta-\lambda)}{\sqrt{2 \pi|\delta-\lambda|}|\kappa|}\left[\cos (\delta) C\left(\sqrt{\frac{2|\delta-\lambda|}{\pi}}\right)+\operatorname{sign}(\delta-\lambda) \sin (\delta) S\left(\sqrt{\frac{2|\delta-\lambda|}{\pi}}\right)\right] \\
-\frac{\operatorname{sign}(\delta-\lambda)}{|\kappa|}[\cos (\delta) \cos (|\delta-\lambda|)+\operatorname{sign}(\delta-\lambda) \sin (\delta) \sin (|\delta-\lambda|)] \\
+\frac{1}{|\kappa|}[\cos (\delta) \cos (\delta-\lambda)+\sin (\delta) \sin (\delta-\lambda)] .
\end{array}
$$

After the initial values of $G$ and $G^{\prime}$ have been calculated, reduce $\lambda$ by the ratio of $\frac{G}{G^{\prime}}$ (Eq. 3.14) and use the new $\lambda$ to calculate new values for $G$ and $G^{\prime}$. Finally, repeat the process. With each iteration $G \rightarrow 0$ and $\lambda$ approaches the root of the function. Figure 3.6 shows a flowchart representation of the algorithm.

$$
\lambda=\lambda-\frac{G}{G^{\prime}}
$$

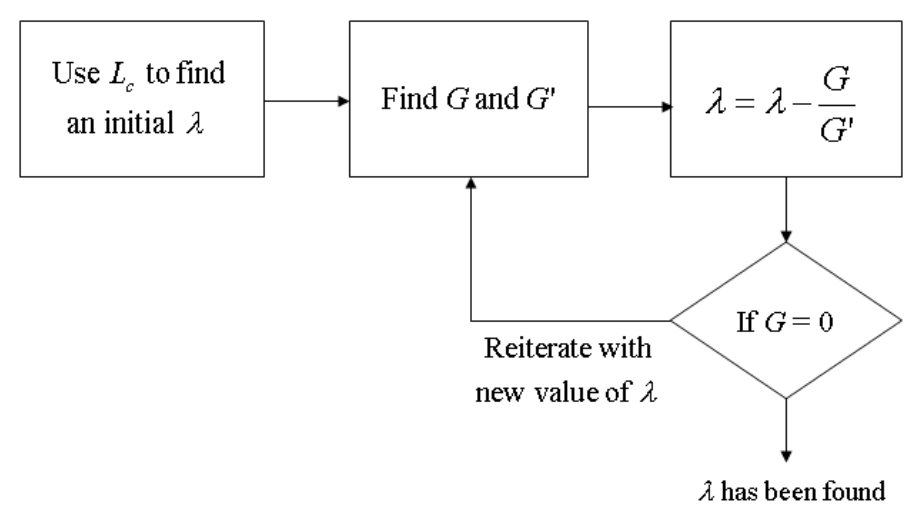

Figure 3.6: Algorithm for finding half of the angle of an inserted arc, $\lambda$, using Newton's root approximation.

Although the root finding method works well, sometimes a zero is not found and $\lambda$ does not converge. When $\lambda$ is non-convergent it does not mean a zero is non-existent or even that the initial guess was wrong. Rather, the distance between the two points is close enough and the change of heading is large enough, relative 
to the distance between the two points, that a $\lambda$ cannot be found such that an arc can be inserted between two non-zero length clothoids. In the case that $\lambda$ is non-convergent, a maximum number of iterations must be placed on the root finding algorithm, otherwise an infinite loop will result. One of the advantages of the Newton root approximation method is the high accuracy within a few iterations. For the case of the trajectory planner, testing shows that $\lambda$ can be found within 10 iterations to an accuracy of $10^{-6}$. Therefore, the maximum number of iterations need not be very large.

It is important to note that although a value for $\lambda$ may be found, it may not be valid for a particular trajectory and restrictions must be placed to ensure that valid trajectories are generated. Two particular cases are when $\lambda>\delta$ and when $\lambda \approx \delta$ but $\lambda$ is still less than $\delta$. If $\lambda$ is greater than $\delta$, then the change of heading for the clothoid, $\delta-\lambda$, has an opposite sign than $\lambda$, as shown in Fig. 3.7. When $\lambda>\delta$ the total change in heading of the trajectory does not equal the desired change of heading. Thus, $\lambda$ must be restricted to have a value less than $\delta$ with the same sign.

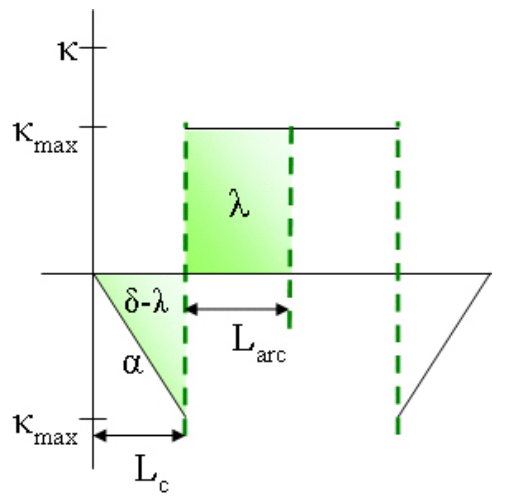

Figure 3.7: Example of the curvature of a trajectory when $\lambda>\delta$.

When $\lambda \approx \delta$ but $\lambda$ is still less than $\delta$, the lengths of the symmetric clothoids are near zero (e.g., $L<=10^{-6}$ ). Because the clothoids have non-zero length the trajectory's curvature is still continuous, however, the trajectory is not drivable (i.e., $\alpha \approx \infty$, meaning the vehicle would need to be stopped in order to turn the steering 
wheel). A restriction must be placed to ensure that clothoids with near zero lengths do not occur in trajectories.

Remember that $\alpha, \kappa, \delta$, and $\lambda$ all effect the clothoid length. However, $\kappa$ is fixed at $\kappa_{\max }$ when inserting an arc and $\delta$ is calculated from the waypoint headings, so neither value can change. Therefore, one of three restrictions could be placed to

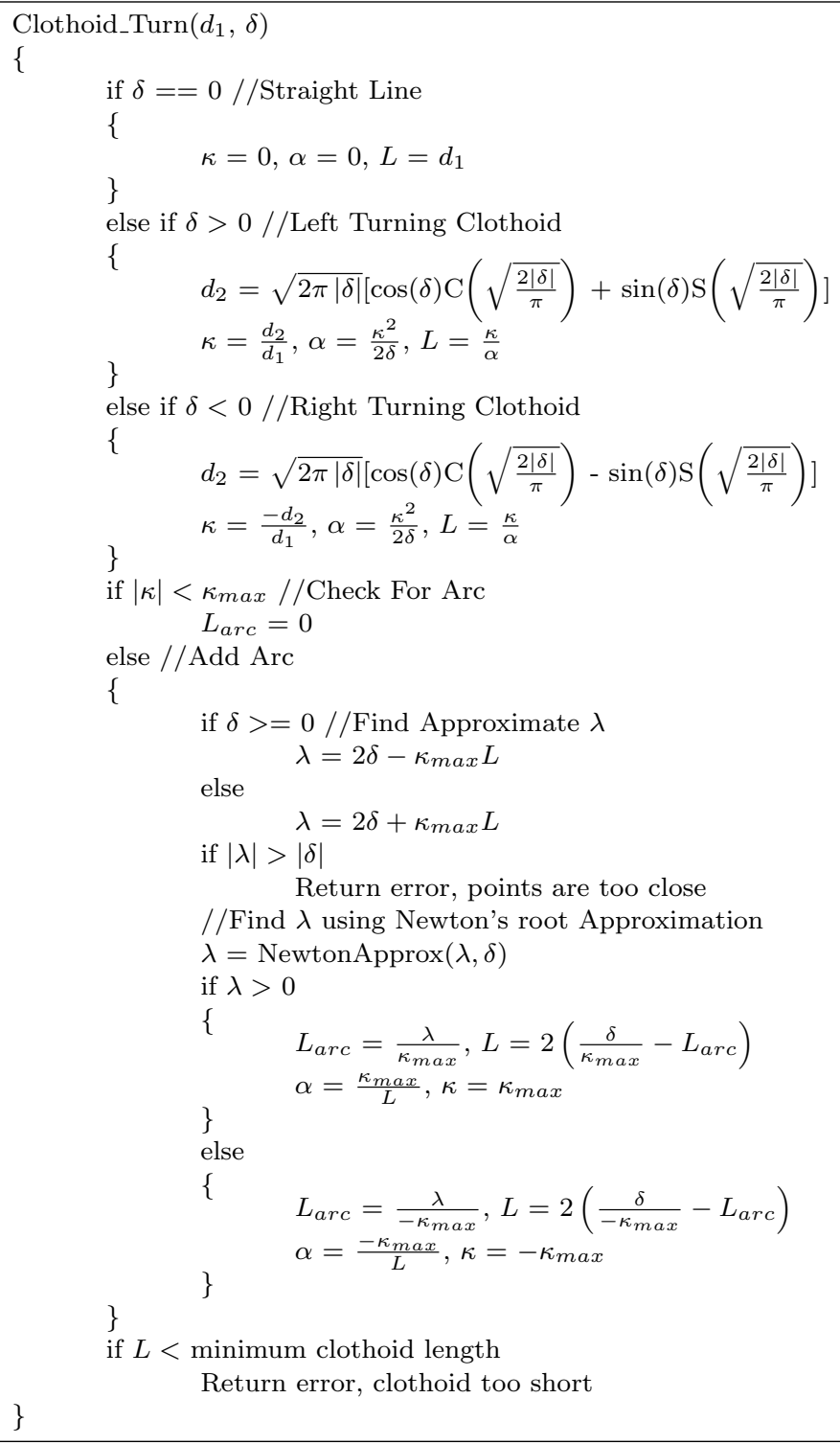

Figure 3.8: Clothoid_Turn() algorithm that includes arcs. Outputs of the algorithm are clothoid curvature, $\kappa$, sharpness, $\alpha$, length of the clothoid, $L$, and length of the arc, $L_{\text {arc }}$. 
ensure that near zero clothoid lengths do not occur in trajectories. Either a minimum clothoid length is determined, or a maximum $\lambda$ or $\alpha$ is found. Finding a maximum $\alpha$ requires either calculating or measure the maximum steering rate of the vehicle and relating the rate to $\alpha$.

Table 3.1: Restrictions placed when adding circular arcs to clothoids.

\begin{tabular}{c|c|c}
\hline \hline Case & Result & Restriction \\
\hline$\lambda>\delta$ & $\operatorname{sign}(\delta-\lambda)=-\operatorname{sign}(\lambda)$ & $\lambda<\delta, \operatorname{sign}(\lambda)=\operatorname{sign}(\delta)$ \\
$\lambda \approx \delta$ & $L_{c} \approx 0$ & $L \geq L_{\min }, \lambda \leq \lambda_{\max }$, or $\alpha \leq \alpha_{\max }$
\end{tabular}

In the case of the motion planner presented in this thesis, it seemed simplest to set a minimum length clothoid that the vehicle could follow rather than calculate $\lambda_{\max }$ or $\alpha_{\max }$. Table 3.1 shows the restrictions in tabular form and Fig. 3.8 shows the Clothoid_Turn() algorithm that includes adding circular arcs and restrictions.

\subsection{Conclusion}

Using a combination of straight lines, clothoids, and arcs, trajectories are generated that are smooth, natural, and drivable. Arcs are used to provide an upperbound on curvature such that the maximum curvature of a vehicle is not exceeded. Clothoids are used to make the transitions between straight lines and arcs smooth and drivable. The next step is to create maneuvers from the trajectories to be used by the vehicle. 


\section{Chapter 4}

\section{Application of Trajectories}

\subsection{Introduction}

This chapter discusses some trajectories created for common driving maneuvers of a DARPA Urban Challenge vehicle using clothoid-arc sets and straight lines. Each of the trajectories follow the specifications that a trajectory begins and ends with zero curvature, the vehicle's minimum turning radius (i.e., maximum curvature, $\left.\kappa_{\max }\right)$ is not violated, and the only information given to the system are the initial and final points and their associated headings.

\subsection{Generic Turn Trajectory}

Clothoid sets or clothoid-arc sets can only provide left-turn, right-turn, and uturn (u-turns are discussed in Section 4.3.3) trajectories. However, one of the purposes of the motion planner is to develop paths between any two points and headings provided by the path planner. Because the Clothoid_Turn() algorithm uses only one constructive polyline, it cannot generate maneuvers that require more than one curve between two points. The purpose of the generic turn is to use two constructive polylines to create a trajectory between any two given points with any two headings, assuming there is sufficient distance between the points that a trajectory can be created within the constraints stated in the introduction of this chapter. This section describes how the generic turn algorithm is developed.

Given the total change in heading and the length of line between the two waypoints, the algorithm calculates the length of two constructive polylines and the change of headings associated with the clothoid sets for the two constructive polylines (Fig. 4.1). Then the generic turn algorithm calls the Clothoid_Turn() algorithm for 
each of the two calculated constructive polylines and the change of headings for the clothoid sets.

\subsubsection{Generic Turn Algorithm}

Figure 4.1 shows a generic turn in the $s \kappa$ plane and in the $x y$ plane. To create a trajectory between the initial point $x_{0}, y_{0}$ and the final point $x_{f}, y_{f}$, the lengths of two constructive polylines $a$ and $b$, and the deflection angles $\delta_{0}$ and $\delta_{f}$ of the first and second clothoid-arc sets, respectively, must be computed (Fig. 4.1). Where polylines $a, b$, and line $d$ are the sides of a triangle formed by the initial and final points and a midpoint (Fig. 4.1b). The only input variables of the system are initial and final points $x_{0}, y_{0}, x_{f}$, and $y_{f}$ and their associated headings $\theta_{0}$ and $\theta_{f}$, respectively. From these inputs, values for $d, \psi_{0}, \psi_{f}, \eta$, and $\varphi$ are found using Eqs. 4.4, 4.5, 4.6, 4.1, and 3.1 , respectively. Where $d$ is the length of line between the initial and final points, $\psi_{0}$ is the angle between the heading of the initial point and line $d, \psi_{f}$ is the angle from the same line $d$ and the heading of the final point, $\eta$ is the direction angle between adjacent polylines $a$ and $b$, and $\varphi$ is the angle from the initial point to the final point (along line $d$ ). (It is important to note that half of the lengths of $a$ and $b$ passed to the Clothoid_Turn() algorithm as the contructive polylines to scale clothoids and generate the clothoid sets in Fig. 4.1b.) Using the geometry in Fig. 4.1b, equations for constructive polylines $a$ and $b$ are derived using the Law of Sines. Equations 4.14.6 are also obtained from the figure and are used to compute values for angles $\delta_{0}$ and $\delta_{f}$.

$$
\begin{gathered}
\varphi=\operatorname{atan}\left(\frac{y_{f}-y_{0}}{x_{f}-x_{0}}\right), \\
\theta_{0}=\varphi-B-\delta_{0}, \\
\theta_{f}=\varphi+A+\delta_{f},
\end{gathered}
$$




$$
\begin{aligned}
& \psi_{0}=\varphi-\theta_{0}=\delta_{0}+B, \\
& \psi_{f}=\theta_{f}-\varphi=\delta_{f}+A, \\
& \eta=A+B=\delta_{0}+\delta_{f} .
\end{aligned}
$$

For the first clothoid set in the generic turn, the deflection is labeled as $\delta_{0}$ and the second clothoid set deflection is $\delta_{f}$. Thus, the total deflection from the initial point to the final point is the sum of all angles $\delta_{0}$ and $\delta_{f}$ (Eq. 4.7).

$$
\theta_{f}-\theta_{0}=2\left(\delta_{0}+\delta_{f}\right)=2 \eta .
$$

Because $A, B, \delta_{0}$, and $\delta_{f}$ cannot be computed from Fig. $4.1 \mathrm{~b}$ and Eqs. 4.14.7 , they must be fixed before the construction polylines $a$ and $b$ can be determined. Table 4.1 is used to summarize the constraints of Eqs. 4.2-4.6 that restrict the values for $A, B, \delta_{0}$, and $\delta_{f}$. The sums of rows and columns, $\eta$, and $\psi_{0}, \psi_{f}$, respectively, are functions of inputs in Eqs. 4.1, 4.4, 4.5, and 4.6. The horizontal rows sum to $A+B=\eta$ (Eq. 4.6); $\delta_{0}+\delta_{f}=\eta$ (Eq. 4.6); and $\psi_{0}+\psi_{f}=2 \eta$. The vertical columns sum to $A+\delta_{f}=\psi_{f}$ (Eq. 4.5); $B+\delta_{0}=\psi_{0}$ (Eq. 4.4); and $\eta+\eta=2 \eta$.

Table 4.1: Constraints of Eqs. 4.2-4.6

\begin{tabular}{cc|c}
\hline \hline$A$ & $B$ & $\eta$ \\
$\delta_{f}$ & $\delta_{0}$ & $\eta$ \\
\hline$\psi_{f}$ & $\psi_{0}$ & $2 \eta$
\end{tabular}

While Table 4.1 constrains the variables $A, B, \delta_{0}$, and $\delta_{f}$, there remains one degree of freedom in the system, $A+B=\delta_{0}+\delta_{f}$. To characterize an entire family of solutions, a null space parameter $q$ is introduced. By adding a null space to Table 4.1 it allows $A, B, \delta_{0}$, and $\delta_{f}$, to be adjusted with respect to each other while maintaining 

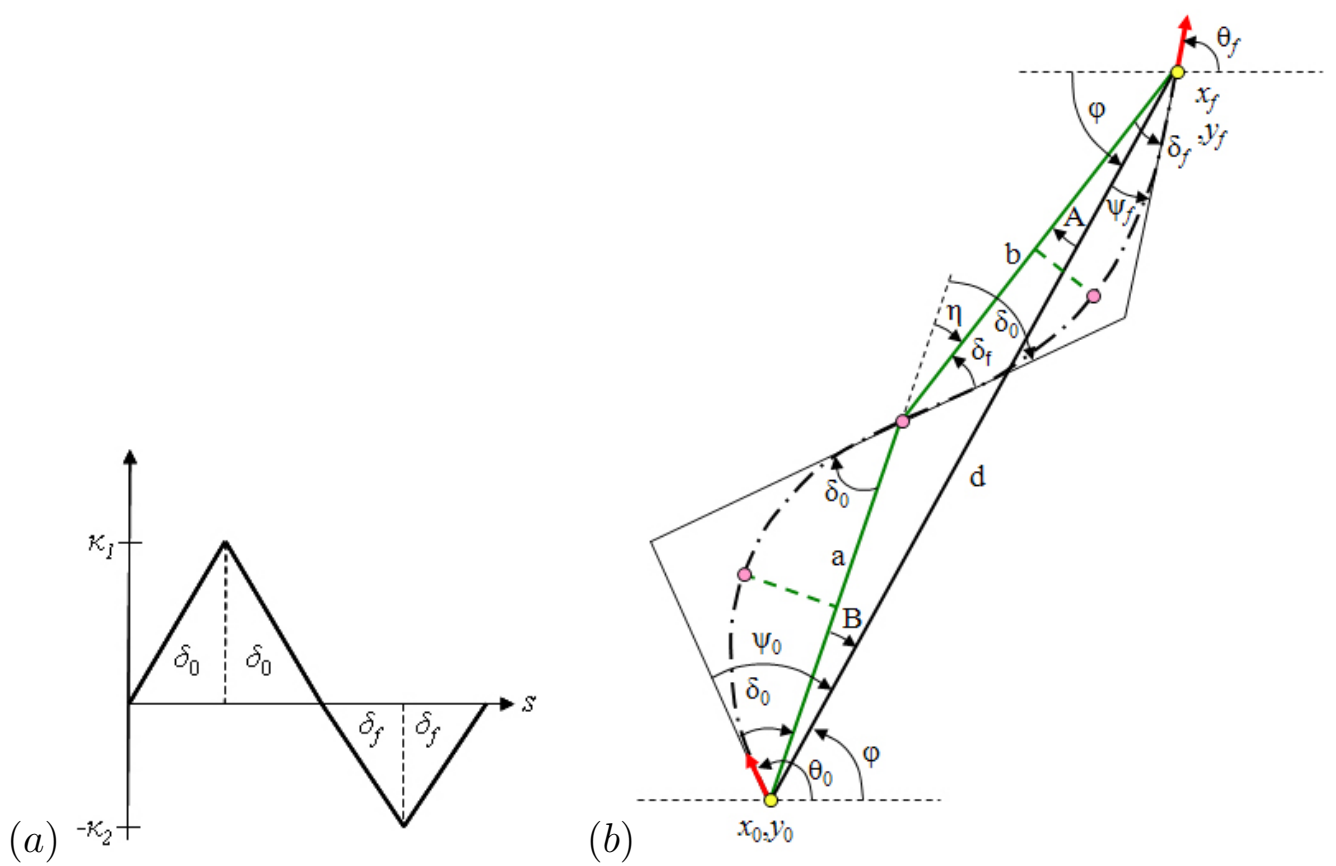

Figure 4.1: Generic turn trajectory in the $(a) s \kappa$ plane and $(b) x y$ plane. (Note that line $d$ here is different from line $d$ in Fig. 3.1b).

the integrity of the constraints. Table 4.2 shows that given a solution $\left(A, B, \delta_{0}, \delta_{f}\right)$ then $\left(A+q, B-q, \delta_{0}+q, \delta_{f}-q\right)$ is also a solution for any value of $q$.

Table 4.2: Generic turn with null space parameter $q$ added to constraints

\begin{tabular}{cc|c}
\hline \hline$A+q$ & $B-q$ & $\eta$ \\
$\delta_{f}-q$ & $\delta_{0}+q$ & $\eta$ \\
\hline$\psi_{f}$ & $\psi_{0}$ & $2 \eta$
\end{tabular}

To show that a null space of Table 4.2 does not change the solution of the constraints, let the constraints of Table 4.1 be put into matrix form (Eq. 4.8).

$$
\left[\begin{array}{llll}
1 & 1 & 0 & 0 \\
0 & 0 & 1 & 1 \\
1 & 0 & 1 & 0 \\
0 & 1 & 0 & 1
\end{array}\right]\left[\begin{array}{c}
A \\
B \\
\delta_{f} \\
\delta_{0}
\end{array}\right]=\left[\begin{array}{c}
\eta \\
\eta \\
\psi_{f} \\
\psi_{0}
\end{array}\right]
$$


The system null space is found to be

$$
\left[\begin{array}{llll}
1 & 1 & 0 & 0 \\
0 & 0 & 1 & 1 \\
1 & 0 & 1 & 0 \\
0 & 1 & 0 & 1
\end{array}\right]\left[\begin{array}{c}
1 \\
-1 \\
-1 \\
1
\end{array}\right]=0 .
$$

Adding the null space, multiplied by parameter q, to the results of Eq. 4.8 (see Eq. 4.10) results in:

$$
\begin{aligned}
& {\left[\begin{array}{llll}
1 & 1 & 0 & 0 \\
0 & 0 & 1 & 1 \\
1 & 0 & 1 & 0 \\
0 & 1 & 0 & 1
\end{array}\right]\left(\left[\begin{array}{l}
A \\
B \\
\delta_{f} \\
\delta_{0}
\end{array}\right]+q\left[\begin{array}{c}
1 \\
-1 \\
-1 \\
1
\end{array}\right]\right)=} \\
& {\left[\begin{array}{llll}
1 & 1 & 0 & 0 \\
0 & 0 & 1 & 1 \\
1 & 0 & 1 & 0 \\
0 & 1 & 0 & 1
\end{array}\right]\left[\begin{array}{l}
A \\
B \\
\delta_{f} \\
\delta_{0}
\end{array}\right]+q\left[\begin{array}{llll}
1 & 1 & 0 & 0 \\
0 & 0 & 1 & 1 \\
1 & 0 & 1 & 0 \\
0 & 1 & 0 & 1
\end{array}\right]\left[\begin{array}{c}
1 \\
-1 \\
-1 \\
1
\end{array}\right]=\left[\begin{array}{c}
\eta \\
\eta \\
\psi_{f} \\
\psi_{0}
\end{array}\right] .}
\end{aligned}
$$

Thus, the free parameter $q$ characterizes an entire family of solutions given valid values for $A, B, \delta_{0}$, and $\delta_{f}$. Although any number of solutions satisfy the equations in Table 4.1, Eq. 4.6 immediately suggests the candidate solutions: ( $A=0$, $B=0),\left(A=0, B=\delta_{0}+\delta_{f}\right),\left(A=\delta_{0}+\delta_{f}, B=0\right),\left(A=\delta_{0}, B=\delta_{f}\right)$, and $\left(A=\delta_{f}\right.$, $B=\delta_{0}$ ). The solution $A=0$ and $B=0$ produces a straight line with a length of $a+b$. The second and third solutions are impossible because they would require that one of the angles of the triangle formed by lines $a b d$, of which $A$ and $B$ are angles of, be zero. Both trajectories are members of the generic turn family and are discussed in Section 4.3.1. This leaves the two candidate solutions: $A=\delta_{0}, B=\delta_{f}$ and $A=\delta_{f}$, $B=\delta_{0}$. By substituting the first remaining candidate solution into Eqs. 4.2-4.6, the following equations are produced. 


$$
\begin{gathered}
\theta_{0}=\varphi-\delta_{0}-\delta_{f}, \\
\theta_{f}=\varphi+\delta_{0}+\delta_{f}, \\
\psi_{0}=\delta_{0}+\delta_{f}, \\
\psi_{f}=\delta_{0}+\delta_{f}, \\
\eta=\delta_{0}+\delta_{f} .
\end{gathered}
$$

Table 4.3 summarizes the constraints for the solution $A=\delta_{0}$ and $B=\delta_{f}$. Summing the columns and rows of the table show that the solution is valid, but only when $\psi_{0}=\psi_{f}$ (Eqs. 4.13 and 4.14). As the matrix of Table 4.1 is rank deficient (Eq. 4.8), one of the dependent rows can be removed and the new constraint, $\psi_{0}=\psi_{f}$, added to form a matrix for Table 4.3.

The constraint $\psi_{0}=\psi_{f}$ added to the other constraints of the table results in a full rank system and is therefore a single point solution.

Table 4.3: Constraints for the solution $A=\delta_{0}$ and $B=\delta_{f}$

\begin{tabular}{cc|c}
\hline \hline$\delta_{0}$ & $\delta_{f}$ & $\delta_{0}+\delta_{f}=\eta$ \\
$\delta_{f}$ & $\delta_{0}$ & $\eta$ \\
\hline$\psi_{f}$ & $\psi_{0}$ & $2 \eta$
\end{tabular}

The next step is to check if the second remaining candidate solution, $A=\delta_{f}$ and $B=\delta_{0}$, is valid and provides a desirable family of solutions. This solution reduces Eqs. 4.2-4.6 to the following equations. 


$$
\begin{gathered}
\theta_{0}=\varphi-2 \delta_{0}, \\
\theta_{f}=\varphi+2 \delta_{f}, \\
\psi_{0}=2 \delta_{0}, \\
\psi_{f}=2 \delta_{f}, \\
\eta=\delta_{0}+\delta_{f} .
\end{gathered}
$$

Table 4.4: Constraints for the solution $A=\delta_{f}, B=\delta_{0}$, and free paramter $q$

\begin{tabular}{cc|c}
\hline \hline$\frac{\psi_{f}}{2}+q$ & $\frac{\psi_{0}}{2}-q$ & $\frac{\psi_{0}+\psi_{f}}{2}=\eta$ \\
$\frac{\psi_{f}}{2}-q$ & $\frac{\psi_{0}}{2}+q$ & $\frac{\psi_{0}+\psi_{f}}{2}=\eta$ \\
\hline$\psi_{f}$ & $\psi_{0}$ & $\psi_{0}+\psi_{f}=2 \eta$
\end{tabular}

Again, Table 4.4 summarizes the constraints for the solution $A=\delta_{f}, B=\delta_{0}$, and free parameter $q$. Each column and row of the table sum correctly, therefore $A=\delta_{f}$ and $B=\delta_{0}$ is a valid solution. Furthermore, there are no other additional constraints introduced to the system and the matrix of Table 4.4 is identical to the matrix of Table 4.1 which is rank deficient with a rank of 3 . Thus, the solution $A=\delta_{f}$ and $B=\delta_{0}$ produces a family of valid solutions for any value of free variable $q$.

It should be noted that $q$ needs to be constrained by $A$ and $B$. As $A$ and $B$ are always the same sign due to the Law of Sines and assuming forward motion trajectories only; thus, $q$ cannot be so large, either positive or negative, as to cause the angles $A+q$ and $B-q$ to have opposite signs. Therefore, when $A+q \geq 0$ and 
$B-q \geq 0$ then $q$ is constrained by $B \geq q \geq-A$. And when $A+q \leq 0$ and $B-q \leq 0$, then $q$ is constrained by $-A \geq q \geq B$.

Finally, after determining a valid solution for $A$ and $B$ and using Eqs. 4.18 and $4.19, \delta_{0}$ and $\delta_{f}$ can be computed, and therefore polylines $a$ and $b$ can be found for each solution within the solution set using the Law of Sines (Eqs. 4.21 and 4.22) on triangle $a b d$ in Fig. 4.1b. With the length of constructive polylines $a$ and $b$ computed, the generic turn problem reduces to two clothoid set trajectories. Figure 4.2 outlines the algorithm for the generic turn.

$$
\begin{gathered}
a=\frac{d \sin |A|}{\sin |\pi-\eta|}=\frac{d \sin \left|\frac{\psi_{f}}{2}+q\right|}{\sin |\eta|} \\
\text { and } b=\frac{d \sin |B|}{\sin |\pi-\eta|}=\frac{d \sin \left|\frac{\psi_{0}}{2}-q\right|}{\sin |\eta|} .
\end{gathered}
$$

\section{Find $q$ for Generic Turn}

Now that that generic turn algorithm has been defined and $q$ constrained, it must be determined what values of $q$ should be passed to the algorithm. One solution is to let polylines $a=b$ and find the value of $q$ that is valid for $a=b$. Once again, it should be noted that letting $a=b$ is but one solution and other ratios are also valid. However, letting $a=b$ ensures that if one polyline is long enough to create a clothoid-arc set the other polyline is too. Thus, by setting Eqs. 4.21 and 4.22 equal to each other, they reduce to

$$
\sin \left|\frac{\psi_{f}}{2}+q\right|=\sin \left|\frac{\psi_{0}}{2}-q\right| .
$$

To solve for $q$, the arcsine is taken on both sides,

$$
\operatorname{asin}\left(\sin \left|\frac{\psi_{f}}{2}+q\right|+n 2 \pi\right)=\operatorname{asin}\left(\sin \left|\frac{\psi_{0}}{2}-q\right|+m 2 \pi\right)
$$

where $n$ and $m$ are integers. Equation 4.24 then reduces to 


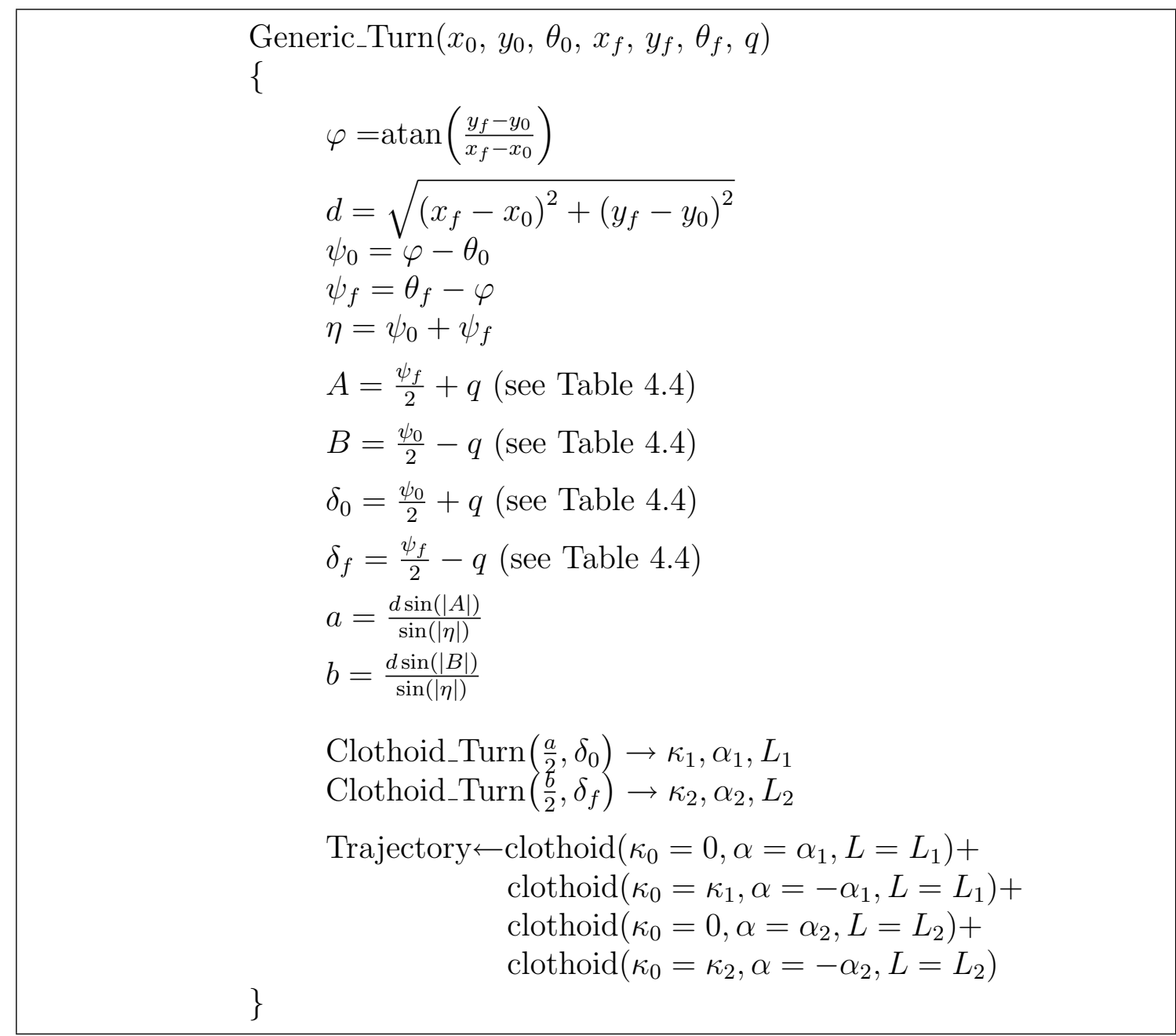

Figure 4.2: Algorithm for a symmetric generic turn trajectory. The output of the generic turn algorithm is a trajectory of four concatenated clothoid turns.

$$
\left|\frac{\psi_{f}}{2}+q\right|+n 2 \pi=\left|\frac{\psi_{0}}{2}-q\right|+m 2 \pi .
$$

Due to the nature of absolute values there are two solutions to the equality, Eqs. 4.26 and 4.27 .

$$
-\frac{\psi_{f}}{2}-q+n 2 \pi=\frac{\psi_{0}}{2}-q+m 2 \pi \rightarrow-\frac{\psi_{f}}{2}=\frac{\psi_{0}}{2}+n^{\prime} 2 \pi
$$




$$
\text { and } \frac{\psi_{f}}{2}+q+n 2 \pi=\frac{\psi_{0}}{2}-q+m 2 \pi \stackrel{\text { Solve for } q}{\longrightarrow} q=\frac{\psi_{0}-\psi_{f}}{4}+n^{\prime} \pi
$$

where $n^{\prime}=m-n$.

Interestingly, the solution in Eq. 4.26 does not contain the parameter $q$. This is the case of a lane change which is discussed in Section 4.3.2. The second solution, Eq. 4.27, provides an equation for $q$ for when polylines $a$ and $b$ are equal. The value of $q$ is calculated and passed to Generic_Turn().

\subsection{Special Case Maneuvers}

The DARPA Urban Challenge competition requires that an autonomous vehicle perform lane changes and u-turns [12]. This section shows how to create specific maneuvers, lane change, curve-straight, straight-curve, and u-turns for an Urban Challenge vehicle.

\subsubsection{Trajectories for Turns and Straight Segments}

Sometimes a clothoid set produces a trajectory that, although valid, is undesirable, such as in the case of making a shallow left-turn at an intersection. For example, if the vehicle on the left in Fig. 4.3a stops at the left part of the intersection, the motion planner should not plan a trajectory such that the autonomous vehicle at the bottom of the figure collides with the vehicle on the left. For these types of situations, it is easier to have special case trajectories that have a straight line either before or after a clothoid set. Figure $4.3 \mathrm{~b}$ shows that the vehicle on the left can be avoided by added a straight line after a clothoid set left-turn.

The generic turn method can produce a straight segment along polyline $a$, followed by one clothoid set using polyline $b$ (Fig. 4.4a) by choosing a specific member of the solution set (i.e., by choosing $q$ ). This is called a straight-curve trajectory and it is produced by setting $q=\frac{-\psi_{0}}{2}$. Applying $q$ to the generic turn, Table 4.4, results in a straight-curve trajectory, Table 4.5, where $\delta_{0}=0$. 

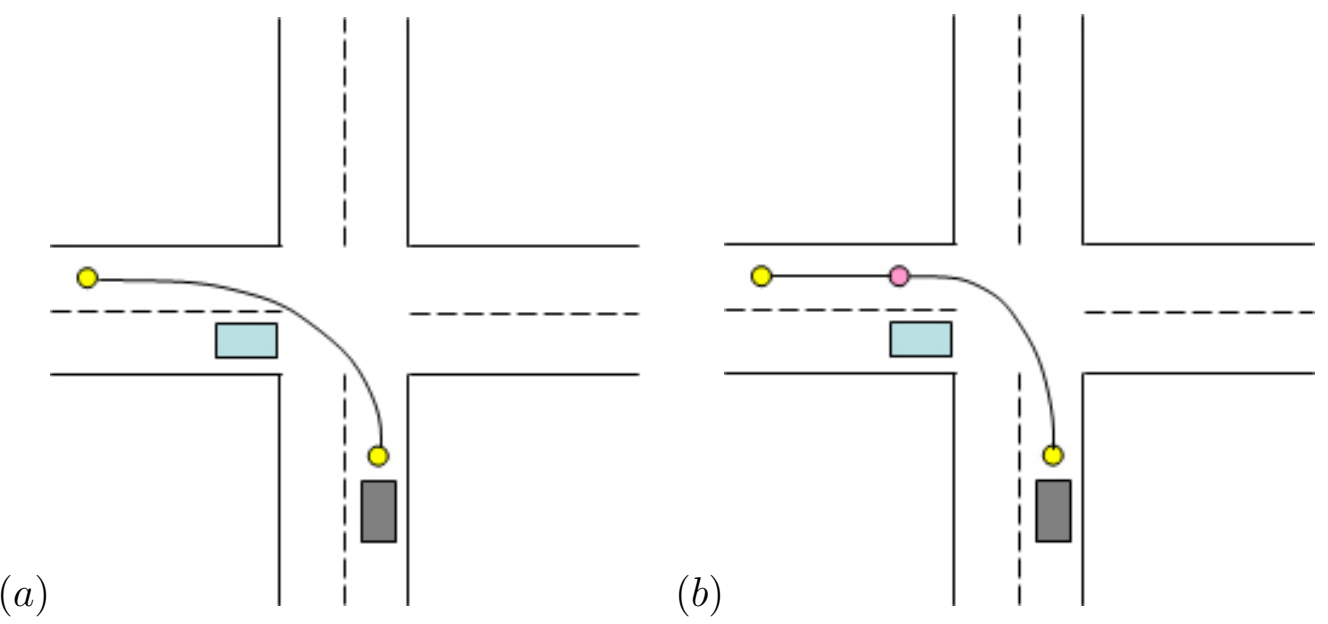

Figure 4.3: (a) Undesirable shallow left-turn with a possible collision. (b) A left-turn consisting of a curve and a straight segment to avoid a collision.

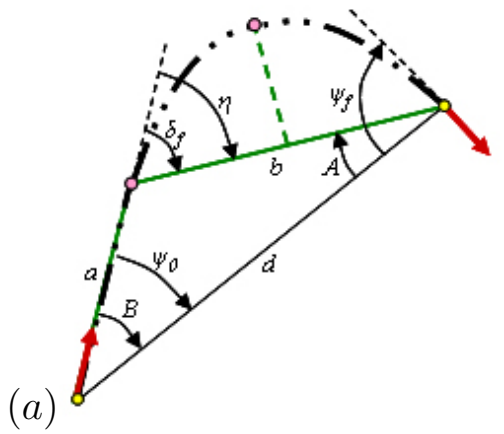

(b)

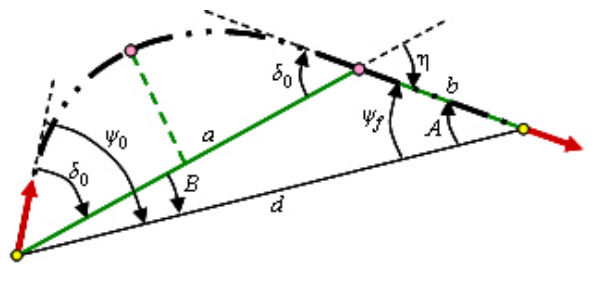

Figure 4.4: (a) Straight-curve trajectory. (b) Curve-straight trajectory.

The generic turn method can also produce a trajectory consisting of a clothoid set on polyline $a$, followed by a straight segment of length $b$ (Fig. 4.4b) by choosing another specific member of the solution set (i.e., by choosing a different $q$ ). This is called a curve-straight trajectory, and is produced by setting $q=\frac{\psi_{f}}{2}$. And again applying $q$ to the generic turn, Table 4.4, results in a curve-straight trajectory, Table 4.6, where $\delta_{f}=0$.

Table 4.5: Constraints for the straight-curve trajectory for $q=\frac{-\psi_{0}}{2}$ (see Table 4.4)

\begin{tabular}{cc|c}
\hline \hline$\frac{\psi_{f}}{2}-\frac{\psi_{0}}{2}$ & $\psi_{0}$ & $\frac{\psi_{0}+\psi_{f}}{2}=\eta$ \\
$\frac{\psi_{f}}{2}+\frac{\psi_{0}}{2}$ & 0 & $\frac{\psi_{0}+\psi_{f}}{2}=\eta$ \\
\hline$\psi_{f}$ & $\psi_{0}$ & $\psi_{0}+\psi_{f}=2 \eta$
\end{tabular}


Table 4.6: Constraints for the curve-straight trajectory

\begin{tabular}{cc|c}
\multicolumn{3}{c}{ for $q=\frac{\psi_{f}}{2}$ (see Table 4.4) } \\
\hline \hline$\psi_{f}$ & $\frac{\psi_{0}}{2}-\frac{\psi_{f}}{2}$ & $\frac{\psi_{0}+\psi_{f}}{2}=\eta$ \\
0 & $\frac{\psi_{0}}{2}+\frac{\psi_{f}}{2}$ & $\frac{\psi_{0}+\psi_{f}}{2}=\eta$ \\
\hline$\psi_{f}$ & $\psi_{0}$ & $\psi_{0}+\psi_{f}=2 \eta$
\end{tabular}

Because the solutions of the maneuvers are members of the solution set of the generic turn, the algorithms for the straight-curve and curve straight maneuvers consist of a single call to Generic_Turn(), passing the appropriate value of $q$.

\subsubsection{Lane Change Trajectory}

One of the requirements of the DARPA Urban Challenge is that the vehicle must be able to pass another vehicle in the same lane if the second vehicle is stopped [12]; the lane change maneuver does just that. The trajectory is used when the beginning and ending point headings are the same (i.e., $\theta_{0}=\theta_{f}$ ) but the points are offset by some $x$ and $y$ distance such that the vehicle can switch lanes on a roadway (Fig. 4.5). This maneuver allows the path planner to provide two waypoints with the same headings. No change of heading means that $\eta=0$. When $\eta=0$, the Law of Sines cannot be used to calculate $a$ and $b$ due to division by zero.

Therefore, while $q$ is passed to the Generic_Turn(), it is ignored for the case of the lane change and an exception must be made in the Generic_Turn() algorithm to handle the division by zero case. To avoid this it is assumed that $a=b=\frac{d}{2}$. While other distances for the polylines will also be valid, the distance of half of $d$ helps ensure that lengths of polylines $a$ and $b$ are sufficiently large enough to create trajectories for them.

Because $\eta=0$, the triangle formed by lines abd disappears. This then changes the angles that are passed to Generic_Turn(). As $A+q$ and $B-q$ are angles in that triangle, then $A+q=B-q=0$. This means that $q$ is ignored, and from Table 4.7 it can be seen that $\delta_{0}=\psi_{0}$ and $\delta_{f}=\psi_{f}$. Figure 4.6 shows the changes made to the Generic_Turn() algorithm to handle the lane change exception. 
Table 4.7: Lane change trajectory constraints for no $q$ and $\theta_{0}=\theta_{f}$ (see Table 4.4)

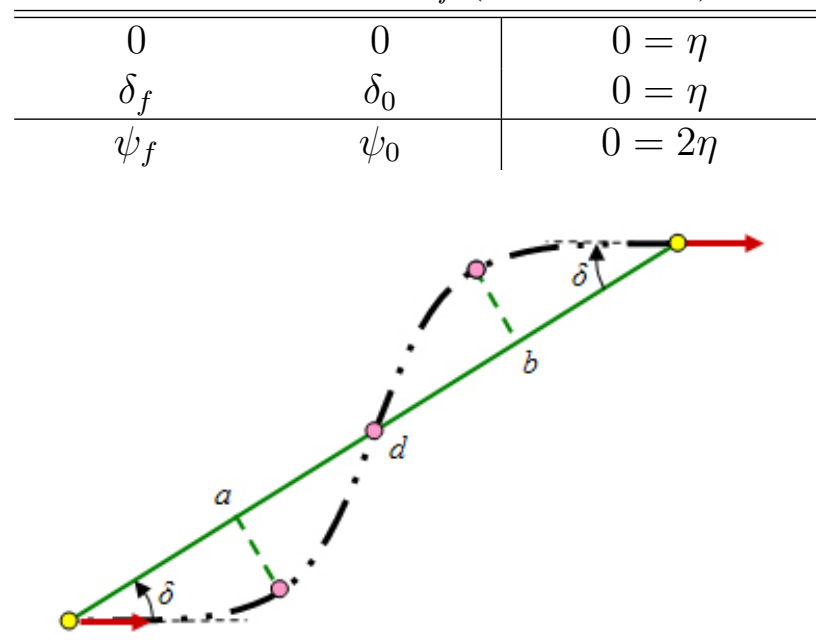

Figure 4.5: Lane change trajectory maneuver

\subsubsection{U-turn Trajectory}

As stated previously, the u-turn is another maneuver required by the Urban Challenge. This trajectory comes about when the total deflection is approximately $\pi$. The actual trajectory is a mirrored clothoid set with $d$ from Eq. 3.1 passed to a single call of Clothoid_Turn(). Only one call to Clothoid_Turn() implies that the u-turn has only one clothoid set, similar to a "C" path by Reeds and Shepp [8]. The trajectory consists of two clothoids (Fig. 4.7a) (i.e., $\operatorname{clothoid}\left(\kappa_{0}=0, \alpha=\alpha, L=L\right)$ $\left.+\operatorname{clothoid}\left(\kappa_{0}=\kappa, \alpha=-\alpha, L=L\right)\right)$ or two clothoids and an arc between them (Fig 4.7b) (i.e., $\operatorname{clothoid}\left(\kappa_{0}=0, \alpha=\alpha, L=L_{c}\right)+\operatorname{arc}\left(\kappa_{0}=\kappa_{\max }, \alpha=0, L=L_{\text {arc }}\right)+$ $\left.\operatorname{clothoid}\left(\kappa_{0}=\kappa_{\max }, \alpha=-\alpha, L=L_{c}\right)\right)$.

\subsection{When to Execute a Trajectory Maneuver}

The motion planner must know how to determine which maneuver to execute. One way is to examine the values and signs of $\psi_{0}, \psi_{f}$, and $\delta$ (as shown in Fig. 4.8). For example, for the maneuvers that have been discussed previously:

- if $\psi_{0}>0$ and $\delta>0$ then a left-turn is performed, 


\begin{tabular}{|c|}
\hline 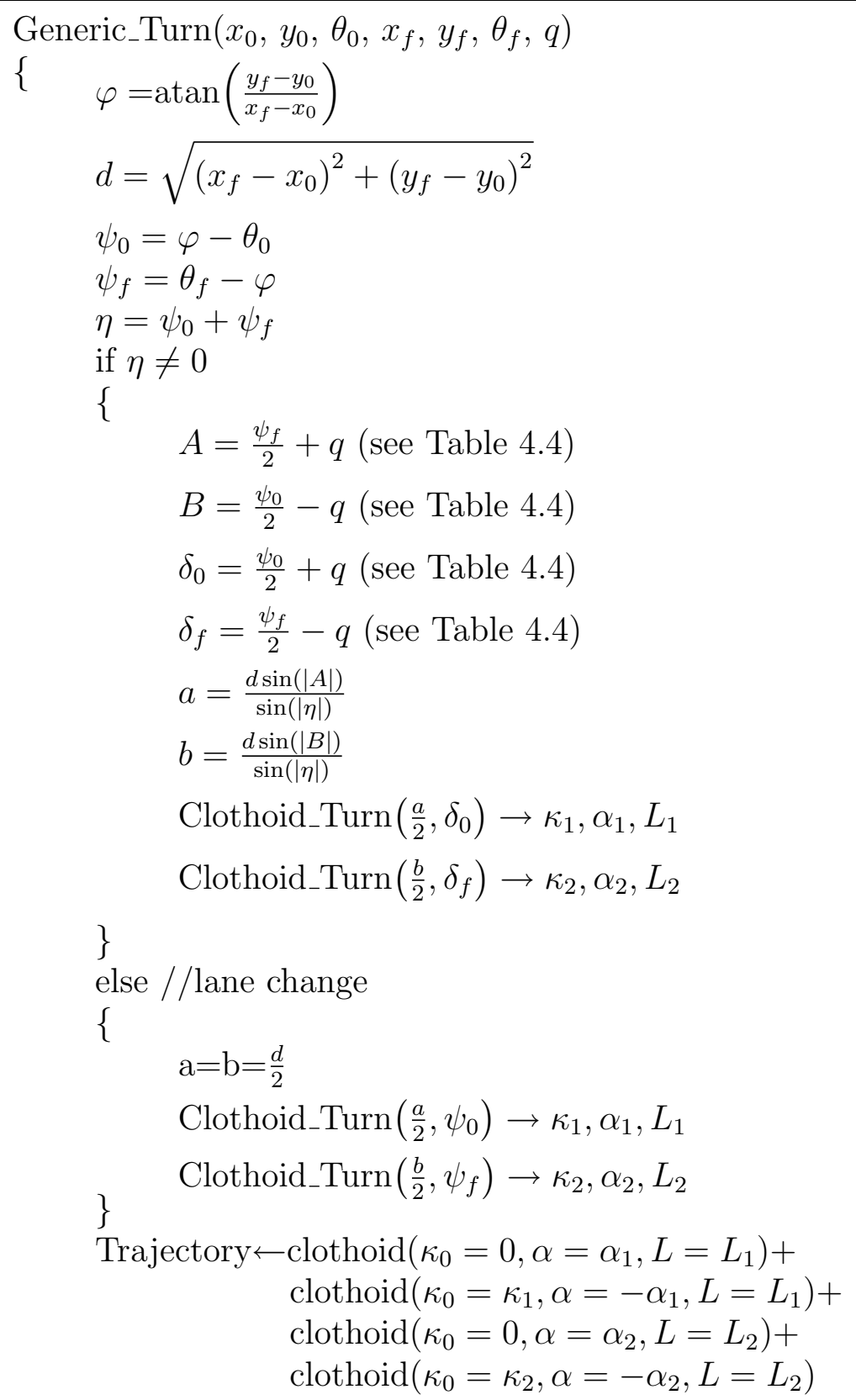 \\
\hline
\end{tabular}

Figure 4.6: Algorithm for a symmetric generic turn trajectory with added lane changes. The output of the algorithm is a trajectory of four concatenated clothoid turns.

- if $\psi_{0}<0$ and $\delta<0$ then a right-turn is performed,

- if $\delta=0$ then a left or right lane change maneuver is executed depending on the sign of $\psi_{0}$, 
(a)

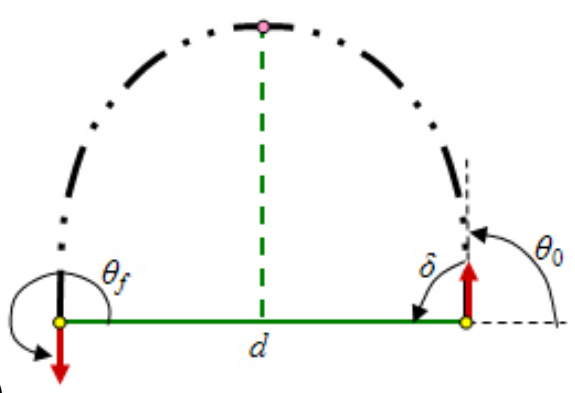

Figure 4.7: (a) U-turn trajectory. (b) U-turn trajectory with an arc.

- if $\psi_{0}=0$ and $\operatorname{sign}\left(\psi_{0}\right)=\operatorname{sign}\left(\psi_{f}\right)$ then a curve-straight maneuver is performed; left or right turn depending on the sign of $\psi_{f}$,

- if $\psi_{f}=0$ and $\operatorname{sign}\left(\psi_{0}\right)=\operatorname{sign}\left(\psi_{f}\right)$ then a straight-curve maneuver is performed; left or right turn depending on the sign of $\psi_{0}$,

- if $\psi_{0}=\frac{\pi}{2}$ and $\delta=\pi$ then a left u-turn is executed.

It is important to note that the feasibility of a maneuver depends on the length of $d$. If $d$ is too small, then it might not be possible to perform a given maneuver. (Imagine taking a u-turn to a point only 2 feet away.) Maneuvers depend upon $d$, $\psi_{0}$, and $\delta$. It seems likely that the minimum length of $d$, for which a maneuver is

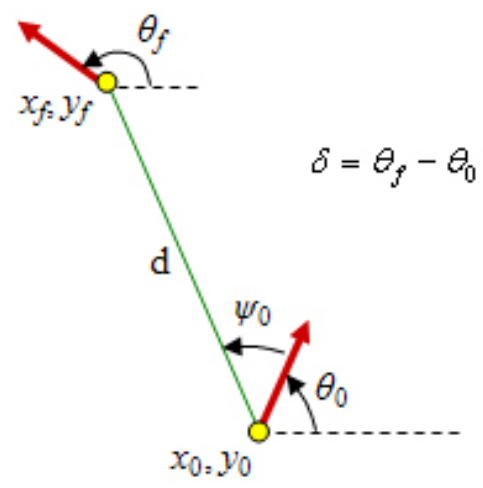

Figure 4.8: Using $\psi_{0}$ and $\delta$ to determine which maneuver to execute. 
feasible (Eq. 4.28), is a function of $\psi_{0}$ and $\delta$. This function is not given here, but it is a matter of future research.

$$
d \geq f\left(\delta, \psi_{0}\right)
$$

Maneuvers that cannot be performed are deemed "Illegal" meaning that either (1.) a two-polyline trajectory for that maneuver cannot be created that is smooth and drivable, (2.) the maneuver is not usually legal or wise to perform in normal traffic situations (e.g., a right hand u-turn), or (3.) the maneuver is not simple and may require driving in reverse (e.g. a 3-point turn).

A further advantage of analyzing the legality of maneuvers is that it adds another check between the path planner and motion planner for drivability and safety. Should the motion planner encounter an illegal maneuver condition, it returns an error to the path planner stating that the motion planner cannot create a safe and drivable trajectory.

\subsection{Results}

The motion planner generates trajectories with the smallest curvature required to create a path between two waypoints. As stated earlier, trajectories generated by the motion planner are similar to paths that a human would drive and are unlike the Fraichard and Scheuer paths that have maximum sharpness and curvature [6]. As there is no standard metric to compare trajectories, one is proposed. The proposed metric is to compare the maximum curvature of a trajectory generated by both planners. The reason that this metric is reasonable is that we humans tend to turn the steering wheel of a vehicle as little as possible. For example, as the distance between two waypoints increases, humans turn the steering wheel less and less turning results in a smaller maximum curvature along a trajectory. Remember, that curvature is the inverse of the radius of the turn circle. 
Also, as the curvature decreases, the centrifugal force on the vehicle and on any on board passengers decreases (Eq. 4.29).

$$
f \propto \kappa v^{2}
$$

where $v$ is velocity of the vehicle or passenger and $f$ is the centrifugal force. A smaller centrifugal force as a result of smaller curvature along a trajectory, not only allows for more passenger comfort (remember the teenager who always turns at maximum curvature) but also for higher velocities along a trajectory. Thus, the drivability and naturalness of the trajectories generated by both planners can be quantized by plotting the metric of maximum curvature along a trajectory with respect to the distance between the beginning and ending waypoints.

Table 4.8: The distance, $d$, between the origin and each of the end waypoints, $x_{f}, y_{f}$, in Fig. 4.9.

\begin{tabular}{c|c} 
End waypoint: $x_{f}, y_{f}$ & $d$ \\
\hline \hline$-6,6$ & 8.49 \\
\hline$-6,10$ & 11.66 \\
\hline$-10,6$ & 11.66 \\
\hline$-10,10$ & 14.14 \\
\hline$-10,15$ & 18.03 \\
\hline$-15,15$ & 21.21 \\
\hline$-15,20$ & 25.0 \\
\hline$-20,20$ & 28.28
\end{tabular}

To obtain data for the metric, the maximum curvature of eight different leftturn trajectories is calculated for both the motion planner and the Fraichard and Scheuer method. The distance, $d$, between the origin and each of the end waypoints is shown in Table 4.8 and the waypoints for the trajectories are shown in Fig. 4.9. The left-turn trajectories all start at the origin and end at one of the other eight waypoints. The trajectories can all start at the origin without loss of generality. The maximum curvature of the DARPA Urban Challenge vehicle for which the motion planner was 


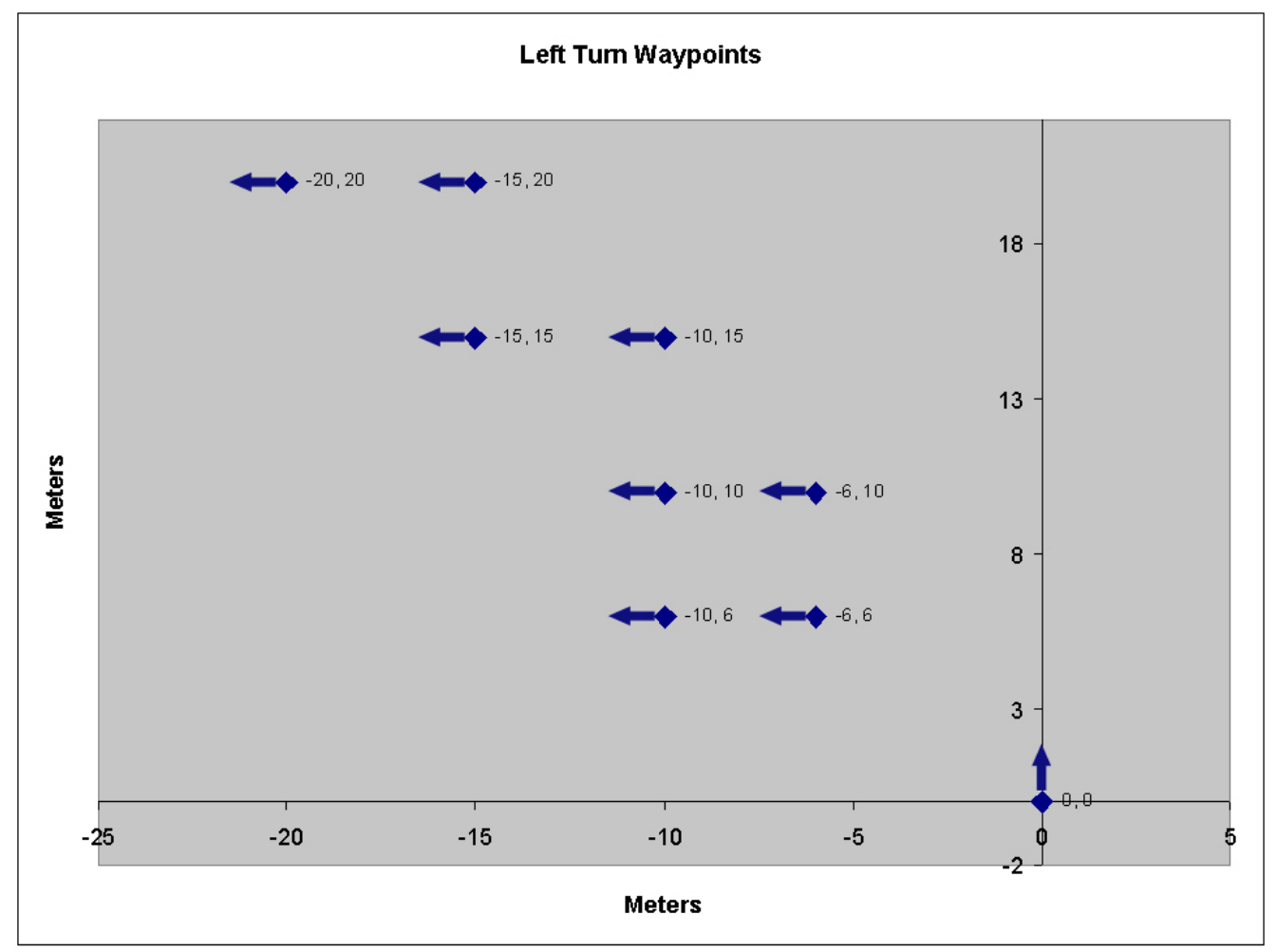

Figure 4.9: Chosen waypoints for left-turn trajectories. Each left-turn starts at the origin and ends at one of the waypoints.

designed, is $0.2 \frac{1}{m}$. Thus, the maximum curvature of both motion planning methods for this vehicle is $0.2 \frac{1}{m}$ and is used as a basis for comparing the two methods.

The maximum curvatures of each left-turn trajectory versus the distance $d$ of each left-turn trajectory for the motion planner and the Fraichard and Scheuer method are shown in Fig. 4.10. Remember, the Fraichard and Scheuer trajectories always have maximum curvature and sharpness, regardless of the type of trajectory. Thus, as the distance between the waypoints increases, the maximum curvature of each trajectory does not change. However, the motion planner presented in this thesis uses the smallest curvature required to generate a trajectory between two waypoints. Therefore, the maximum curvature of a trajectory decreases as the distance between the waypoints increases. The results in Fig. 4.10 show that the trajectories generated by the motion planner are more drivable and natural than those generated by the Fraichard and Scheuer method as the distance $d$ increases. 


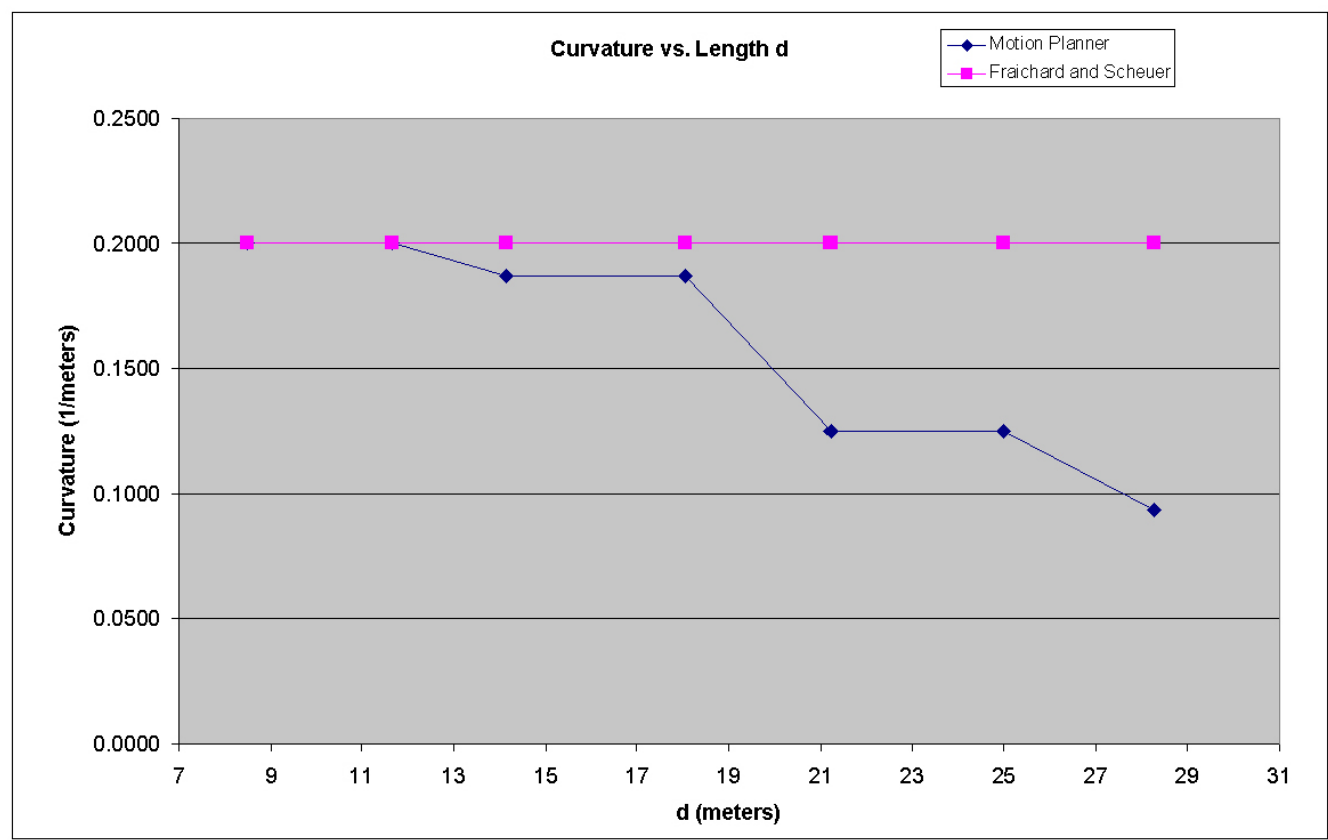

Figure 4.10: Maximum curvature versus length of $d$ for each of the left-turns in Fig. 4.9 for both the motion planner and Fraichard and Scheuer's method.

\subsubsection{Motion Planning a Test Course}

Figure 4.11a shows a test course with hand picked waypoints to use each of the maneuver and trajectory algorithms discussed previously. The motion planner uses each of the maneuvers to plan paths between waypoints in the test course; straight, left-turn, right-turn, curve-straight, straight-curve, left and right lane changes, left u-turn, and the generic turn. The results of the motion planner after planning trajectories between each of the waypoints of the test course are shown in Fig. 4.11b. Two more motion planner results are shown in Figs. 5.6 and 5.8 in Chapter 5.

\subsection{Conclusion}

This chapter has shown how maneuvers are formed from trajectories. An algorithm has been described that creates a trajectory that can begin at a point and heading and end at any point and heading given free variable $q$ and sufficient distance between the points. Three other maneuvers (straight-curve, curve-straight, and lane change) have been discussed and shown to be specific cases of the generic turn. It has 
(a)

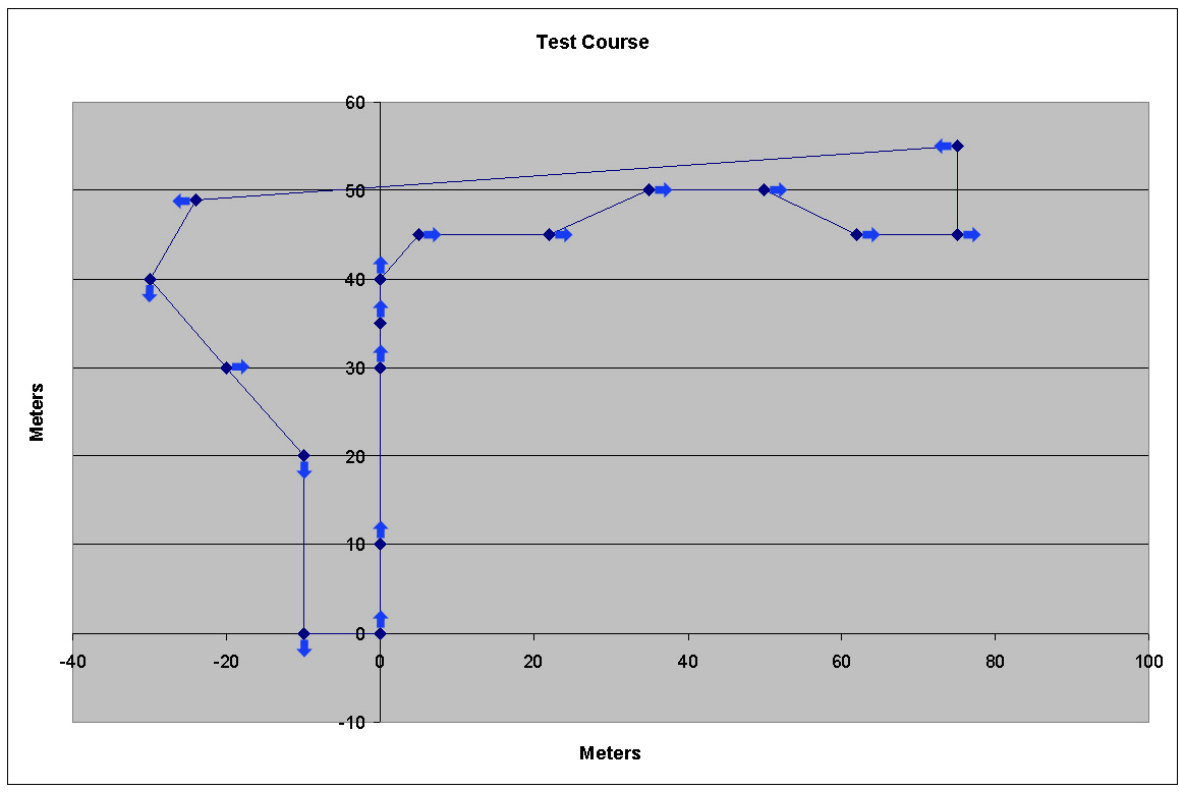

(b)

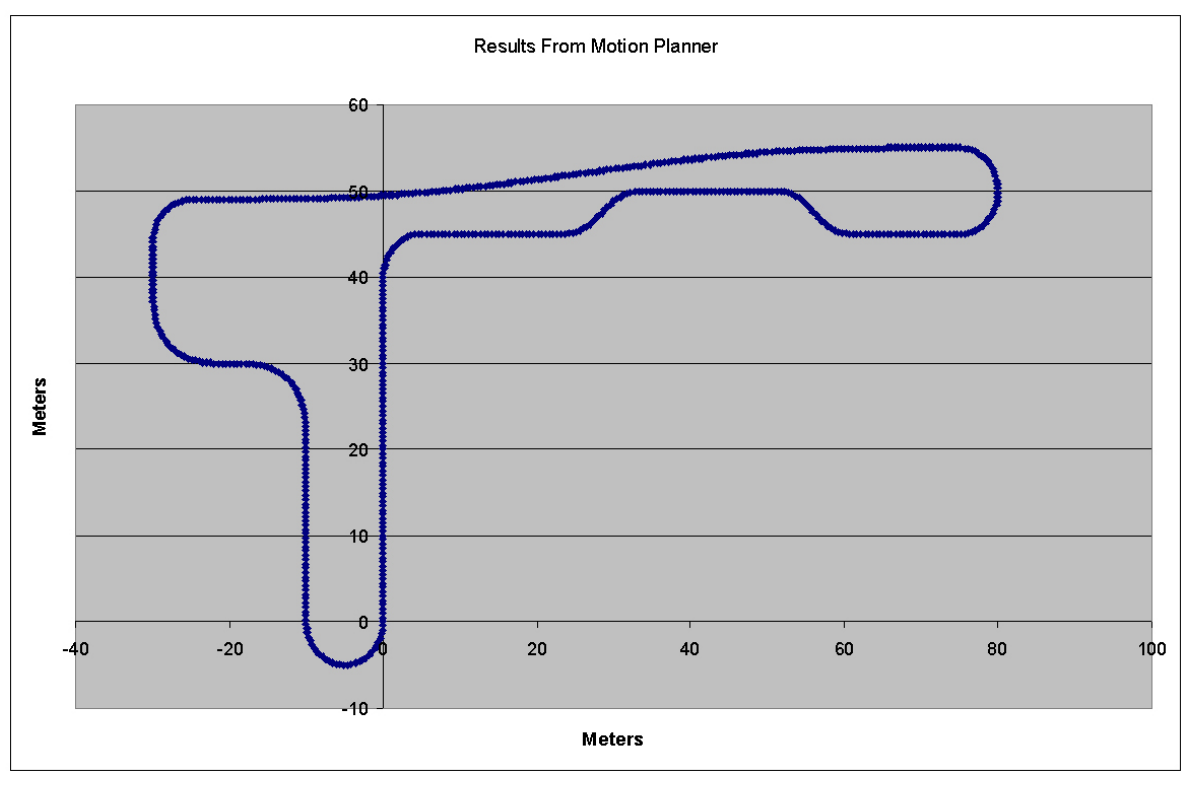

Figure 4.11: (a) Example course with hand picked waypoints. (b) Results of the motion planner after the waypoints are passed to the motion planner. The motion planner uses each of the maneuvers discussed previously to plan the example course; straight, left-turn, right-turn, curve-straight, straight-curve, left and right lane changes, left $\mathrm{u}$-turn, and the generic turn. The trajectories of the motion planner are plotted every 0.25 meters.

also been explained when these maneuvers should be executed. A metric has been proposed and used to compare the motion planner trajectories and the Fraichard and Scheuer trajectories. The results show that the motion planner generates trajectories 
that are more drivable and natural than the Fraichard and Scheuer paths. The next chapter discusses a method of testing the motion planner and shows an example of a planned course. 


\section{Chapter 5}

\section{Testing the Motion Planner}

\section{$5.1 \quad$ Introduction}

As with all software and systems, continuous testing of the motion planner and vehicle system is required throughout all stages of development. A set of waypoints can be used to provide a test course to test the abilities of the motion planner and the vehicle system. For example, Fig. 4.11a is a test course that tests each of the trajectories and maneuvers previously discussed. However, designing a test course by hand picking waypoints to pass to the motion planner is a laborious, time consuming, and sometimes frustrating process. In addition to the difficulty of hand picking waypoints, testing the motion planner on the vehicle is more difficult than using a simulation at a desk. However, it is still important that the trajectories produced by the motion planner be tested on the vehicle at sometime during the development of the vehicle. Therefore, an automated method of designing a test course that can test specific vehicle maneuvers, ensure repeatability, and can be used to test the motion planner and vehicle system both on the vehicle and in simulation is needed.

One way to meet these needs is to record waypoints while manually driving the vehicle in the desired test pattern. Then an automated process, the Point Reduction Algorithm, acts like a path planner and chooses a small set of waypoints from the log file containing the recorded GPS points and vehicle headings for each point. These chosen waypoints make up the test course. The selected waypoints are passed to the motion planner which generates trajectories (test data) between the selected waypoints to recreate the test pattern.

This method can be used to test specific vehicle maneuvers, ensures repeatability (from the same log file), is an automated process, can be used both on the 
vehicle and in simulation, and the course generated by the motion planner or the path driven by the vehicle or simulator can be compared against the manually driven course by overlaying the two courses. The overlay provides a way to visually compare the two courses and identify any discrepancies between them to help in the debugging process. If the motion planner can recreate a path driven by a human driver, it also demonstrates that the motion planner can create drivable and natural paths.

\subsection{Obtaining Control and Test Data}

In order to test the motion planner two types of data are required, control data and test data. The control data is the data that the results of the motion planner are compared against. The test data are the results of the motion planner.

\subsubsection{Control Data}

The control data contains the points and headings of the desired outcome. For example, the vehicle is manually driven in a desired test pattern while recording GPS points and vehicle headings from either the sensor logger or the filtered state logger. The control data (i.e., test pattern; and assuming that the hardware sensors or the Kalman filter work properly), are the desired outcome after the vehicle or simulation is autonomously driven (Fig. 5.1). In other words, a manually driven course provides control data that the autonomously driven vehicle should be able to drive.

\subsubsection{Testing Strategy}

The test data contains waypoints and headings of the output of the motion planner and/or the autonomously driven vehicle or simulation (obtained from the filtered state logger or sensor logger in Fig. 5.1). For example, the motion planner generates trajectories from a few selected waypoints from desired test pattern. The trajectories are passed to the vehicle system which autonomously drives the vehicle while the sensors record GPS points and vehicle headings from either the sensor logger or the filtered state logger. Note, that when recording test data from either the sensor 


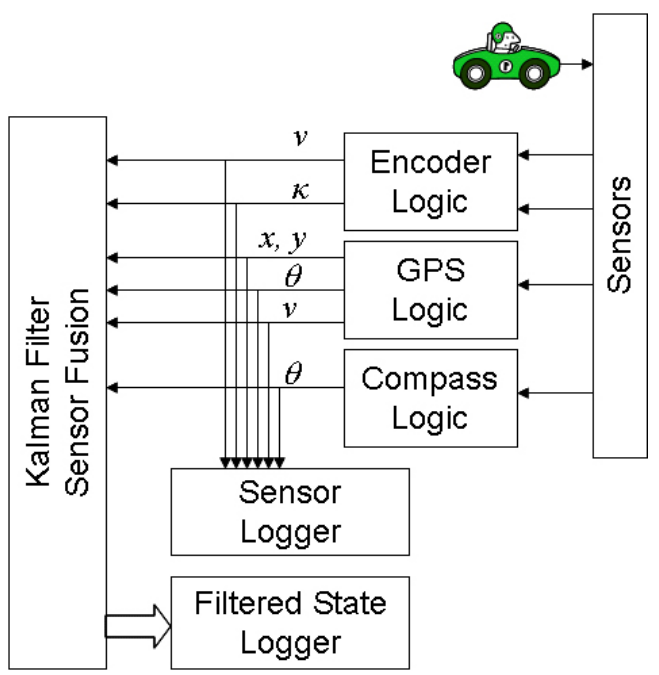

Figure 5.1: Logging control data with the vehicle system while manually driving the vehicle.

logger or the filtered state logger, the same logger should be used in testing that was used when recording the control data.

A successful test would result in a motion planner or vehicle path that matches the control data (i.e., test pattern). An unsuccessful test would result in a significant deviation from the control data. The deviation can help identify components that are not working properly. For example, if the control data takes a right turn and the test data from the motion planner makes a left turn, this signifies that the motion planner fails when creating paths for left and right turns.

\subsection{Point Reduction Algorithm}

The purpose of the Point Reduction Algorithm (PRA) is to provide an automated method of creating a test course by choosing a small number of waypoints from a waypoint log file. The selected waypoints from the log file are then passed the motion planner. The motion planner is tested to see if it can generate trajectories from the selected waypoints that follows or reproduces the test pattern. Thus, the algorithm contains six steps, they are:

1. Read the waypoints from log file 
2. Find the curvature for each waypoint read from the log file

3. Remove all waypoints with non-zero or non-near-zero curvature

4. Pass the remaining waypoints to the Douglas-Peucker algorithm

5. Pass the waypoints selected by the Douglas-Peucker algorithm to the motion planner

6. Choose a new ending waypoint if the motion planner is unable to generate a trajectory

\subsubsection{Finding the Curvature of a Point}

The purpose of calculating the curvature for all of the waypoints is to identify which waypoints have zero or near zero curvature. As all trajectories generated by the motion planner begin and end with no curvature, the points passed to the motion planner by the PRA must have zero or near zero curvature to ensure that the motion planned course properly follows the manually driven course.

After reading in the control data from the log file, the curvature of a waypoint is found by calculating the difference of headings and dividing by the distance between the two points (Fig. 5.2). In other words

$$
\kappa_{x_{i}, y_{i}}=\frac{\theta_{i}-\theta_{i-1}}{\sqrt{\left(x_{i}-x_{i-1}\right)^{2}+\left(y_{i}-y_{i-1}\right)^{2}}} .
$$

It is assumed that the first waypoint, $i=0$, in the control data has zero curvature or at least its curvature is known. This assumption can be made because the initial position and heading of the vehicle can be determined.

Passing only waypoints with zero curvature to the Douglas-Peucker algorithm works well when points exist at all curvature domain inflection points (i.e., there exists a waypoint with zero curvature when the path has zero curvature). The purpose of the Douglas-Peucker algorithm is to reduce the number of points that describe a line [13]. However, points are dropped if there is no waypoint at or very near an inflection 


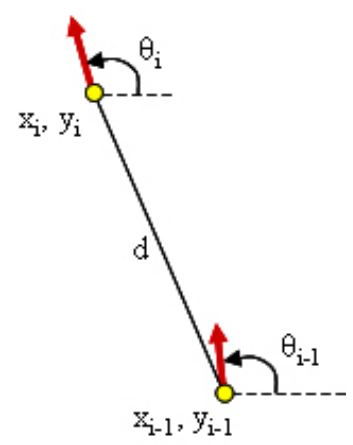

Figure 5.2: Finding the curvature of a waypoint from the headings and the distance between the two waypoints.

point. For example, in Fig. 5.3 all four points would be dropped because they do not have zero or near zero curvature. To ensure that no inflection points are missed, a new waypoint is added when the sign of the curvature changes between two waypoints. The new waypoint is created by taking the average of $x, y$, and $\theta$ of the two waypoints with opposite signs (Eq. 5.2).

$$
\begin{aligned}
& x_{\text {new }}=\frac{x_{i}+x_{i-1}}{2} \\
& y_{\text {new }}=\frac{y_{i}+y_{i-1}}{2} \\
& \theta_{\text {new }}=\frac{\theta_{i}+\theta_{i-1}}{2} .
\end{aligned}
$$

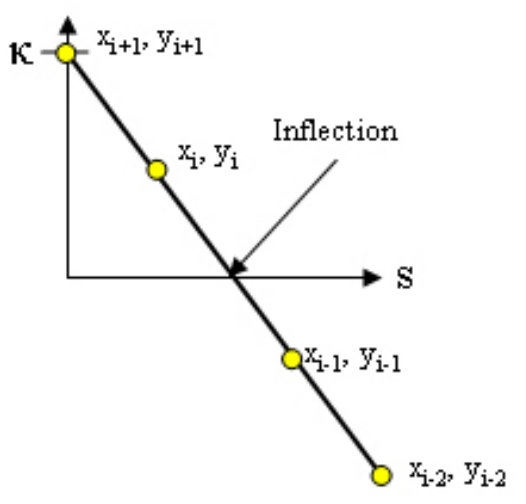

Figure 5.3: All four waypoints would be removed because they do not have zero or near zero curvature. The motion planner would not correctly generate the desired trajectories because of a missing waypoint at the inflection point. A waypoint must be added at the inflection point. 
Then, after calculating the curvature of each point and adding inflection points when needed, all points with non-zero or non-near-zero curvature are removed from the control data. Thus, all the remaining waypoints have no curvature and are then passed to the Douglas-Peucker algorithm which selects a small number of waypoints that describe the test pattern.

\subsubsection{Douglas-Peucker Algorithm}

The log files of the control data contain thousands or even tens of thousands of waypoints. Even after all non-zero and non-near-zero curvature are removed from the control data, the waypoints are too close to each other for the motion planner to generate trajectories between them. As stated previously, the Douglas-Peucker algorithm reduces the number of points that describe a line by selecting the waypoints that fall outside a user-given tolerance.

Because of the algorithm's long history (even as late as the early 2000's the algorithm was still being used in commercial cartography software [14]) and readily available code [15], the algorithm has been shown to select the same number of points as a human would and has very good line generalization [16]. Thus, it was selected to perform the waypoint selection. Despite its strengths, one known drawback to the DP algorithm is that excessively spiky lines are created when used with very large tolerances [16]. To ensure that these spiky lines do not occur, the PRA uses tolerances of approximately half a meter when calling the DP algorithm. Testing has shown that this tolerance level provides good point selection. Some examples are shown in Section 5.4 .

While the DP algorithm was designed to reduce the number of points that describe a line in the spatial domain, it was needed to be able to use waypoint headings in its selection process in order for it to be used by the PRA. While the DP code provided by Sunday [15] was meant to work with the $x, y$, and $z$ axes, the $z$-axis can be used as a heading axis. Meaning, instead of using the $z$-axis as a spatial domain, it can be used as a heading domain. Therefore, the DP code needed to be only slightly modified to accommodate the PRA. 
The DP code was modified to mark the waypoints it selects in the list of waypoints with zero or near zero curvature and to not reduce the list. The reason for marking waypoints and not removing them is because the motion planner may not be able to generate trajectories between the points the DP algorithm selects. Thus, the PRA must accommodate for these cases and choose a new end waypoint by selecting the next waypoint in the list.

\subsubsection{Choosing a New Waypoint}

After the DP algorithm performs waypoint selection, the DP selected waypoints are passed to the motion planner by the PRA. The motion planner's job is to then generate a set of trajectories between the selected waypoints. However, the motion planner is sometimes unable to create a trajectory between the waypoints. When the motion planner can not generate a trajectory, it can be due to an "illegal" maneuver, or any other violations discussed in the previous chapters, such as the waypoints being too close to each other.

When a violation occurs, the PRA must choose a new ending waypoint. The PRA handles a violation by picking the next waypoint from the list of waypoints with zero or near zero curvature, and the new waypoint is passed to the motion planner. If another violation occurs, then the process is iterated until the motion planner can generate a trajectory.

Once the motion planner has generated trajectories for the PRA selected waypoints, the results can be plotted and overlaid with a plot of the control data (i.e., test pattern), allowing the two courses to be compared and examined. Should there be any significant deviation between the two courses, the engineer can use the deviating information to determine what part of the motion planner needs debugging. In addition to testing the motion planner, the PRA can also be used to test the vehicle system. Figure 5.4 shows how the algorithm can be used to autonomously drive the vehicle either in reality or in simulation.

Again, because the control data provides a known desired outcome, should the vehicle or simulation path deviate from the control data, assuming the motion planner 


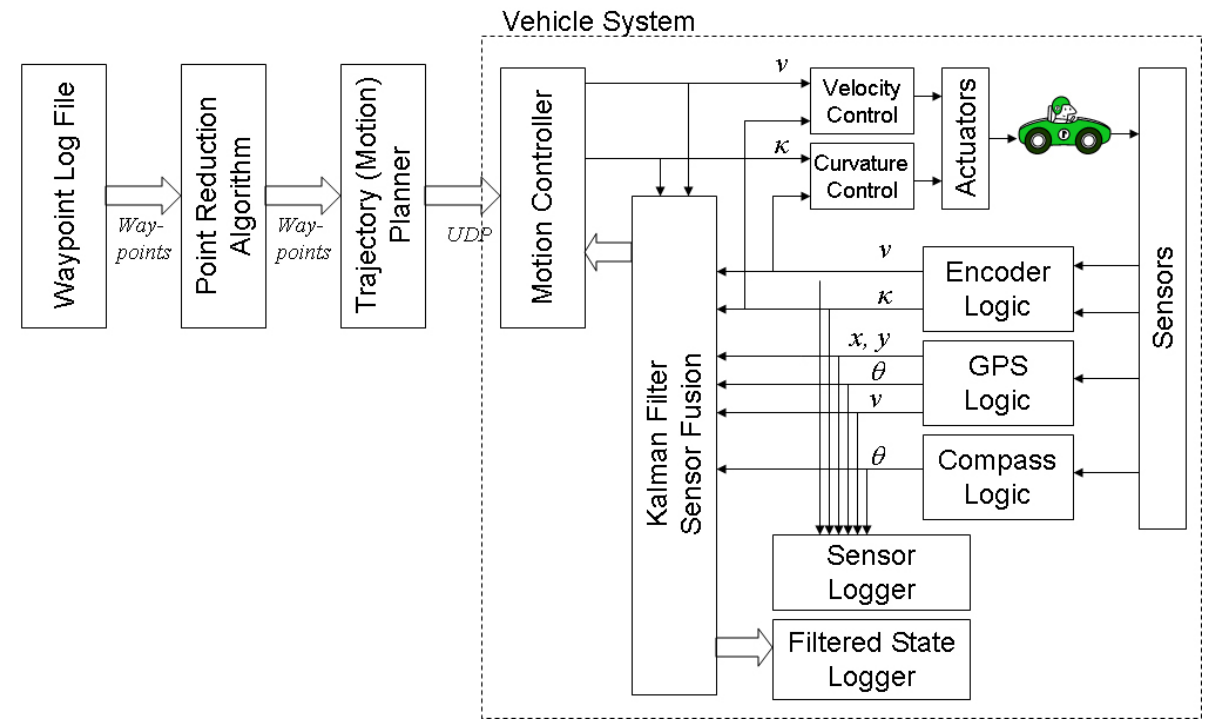

Figure 5.4: Driving the vehicle by reading a log file containing waypoints and using the Point Reduction Algorithm to choose the waypoints and passing them to the motion planner and vehicle controller.

is working properly, system and controller bugs can be determined by comparing the test data with the control data. For example, the vehicle path shows that it is turning late compared to the control data. With further testing and test data collection, the source of the problem can be narrowed and determined.

\subsection{Example Tests of the Motion Planner}

This section shows two examples of testing the motion planner using the PRA. In both examples, the log files contain real control data collected from a minivan using a GPS unit and corrected compass heading. (The compass heading needed to be corrected due to compass lag while turning.) The resulting trajectories from the motion planner in both examples are plotted every 0.25 meters and are overlaid with the control data.

\subsubsection{Test 1}

Figure 5.5a shows an example plot of a log file containing over 2400 waypoints recorded while manually driving the vehicle. The PRA chose only 29 points to de- 


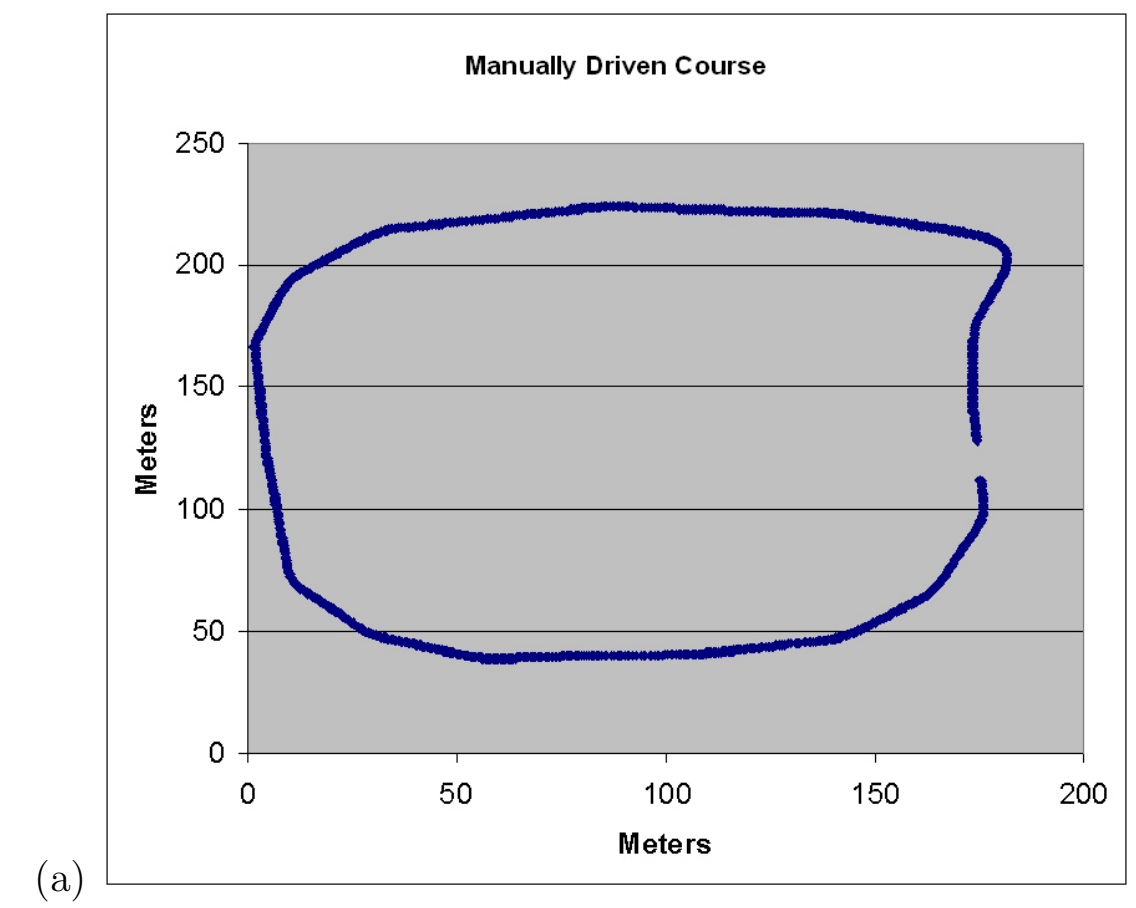

(a)

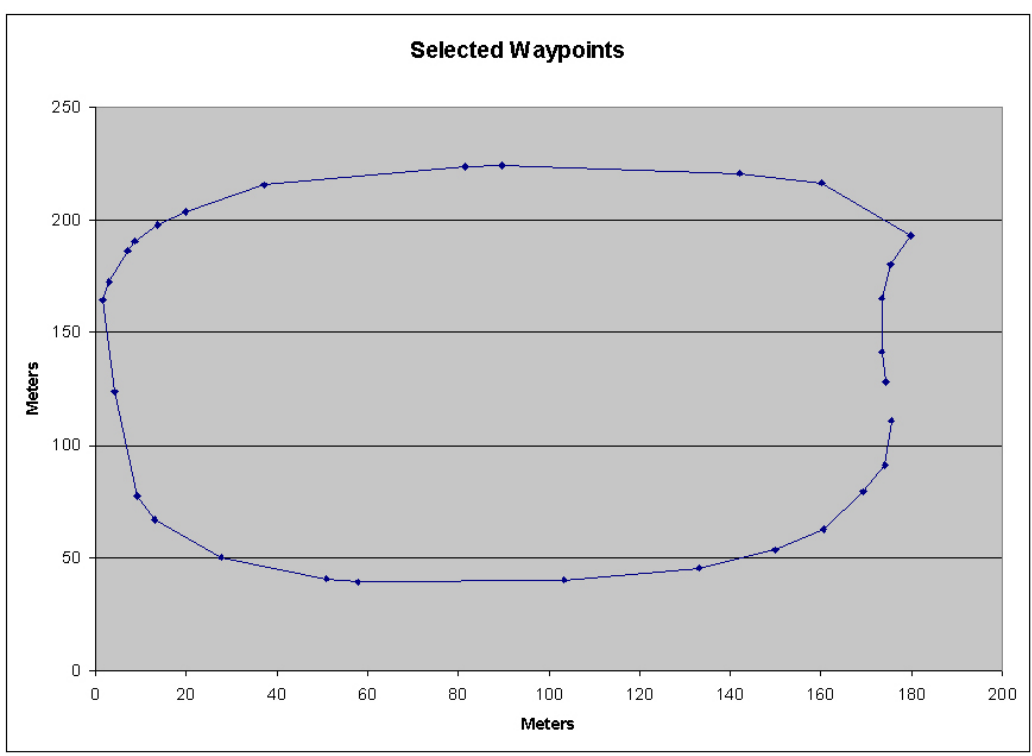

Figure 5.5: (a) A manually driven course with over 2400 waypoints. (b) The waypoints remaining after running the Point Reduction Algorithm on the manually driven course with a DP tolerance of 0.6 meters. These remaining waypoints are passed to the motion planner to create a list of trajectories to recreate the course.

scribe the course, Fig. 5.5b, and then passed these remaining waypoints to the motion planner. Figure 5.6 shows a plot of the results of the path planned by the motion planner with an overlay of the original log file. The results in Fig. 5.6 show that 
given the good input data from Fig. 5.5b, the motion planner provides trajectories that deviate only slightly from the original path.

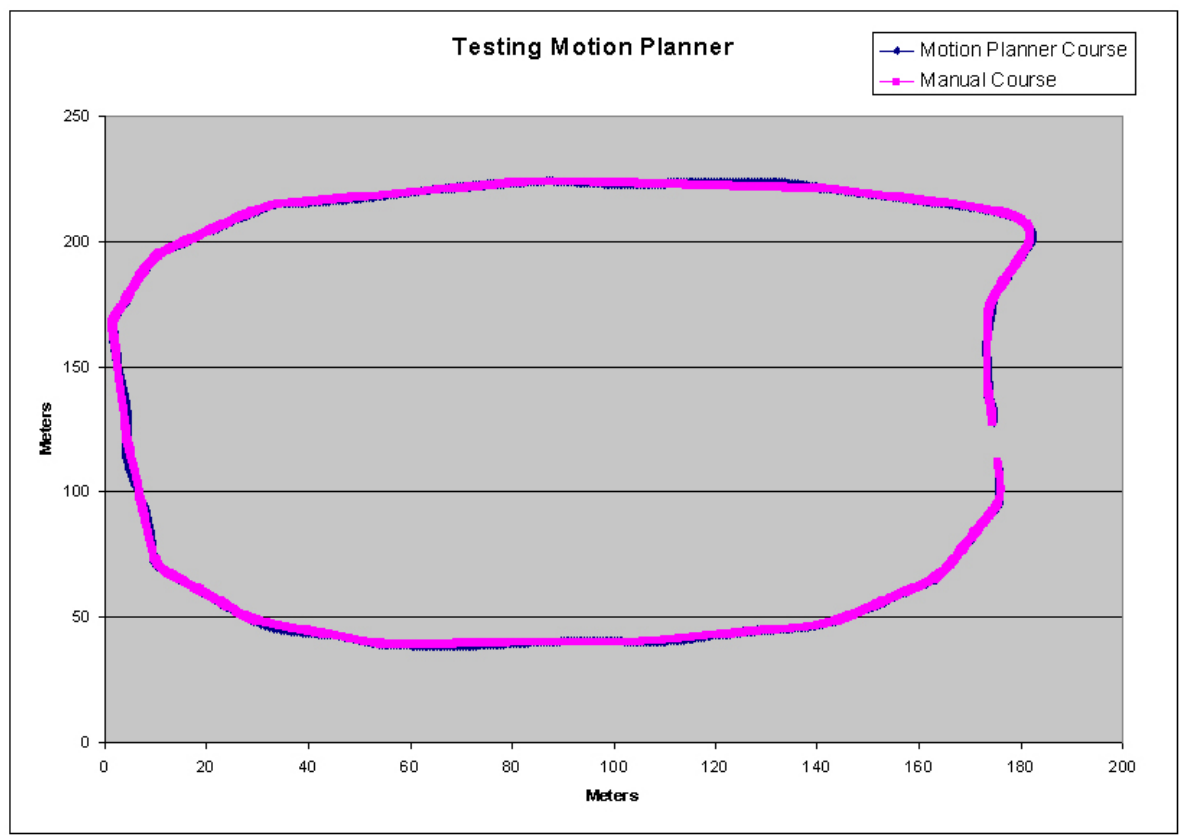

Figure 5.6: Comparison of the first test manual driven course and the course created by the motion planner from the PRA selected points.

\subsubsection{Test 2}

Figure 5.7a shows a second example plot of a log file containing nearly 1000 waypoints recorded while manually driving the vehicle. The PRA chose only 14 waypoints to describe this course, Fig. 5.7b, and passed these remaining waypoints to the motion planner. Figure 5.8a shows a plot of the results of the path planned by the motion planner with an overlay of the original log file.

Unlike the results of the motion planner in the first test in Section 5.4.1, the results of the motion planner in Fig. 5.8a are somewhat poorer, in that the motion planned course significantly deviates from the test pattern. The deviation in the results from the original path could mean that either the motion planner planned a 
(a)

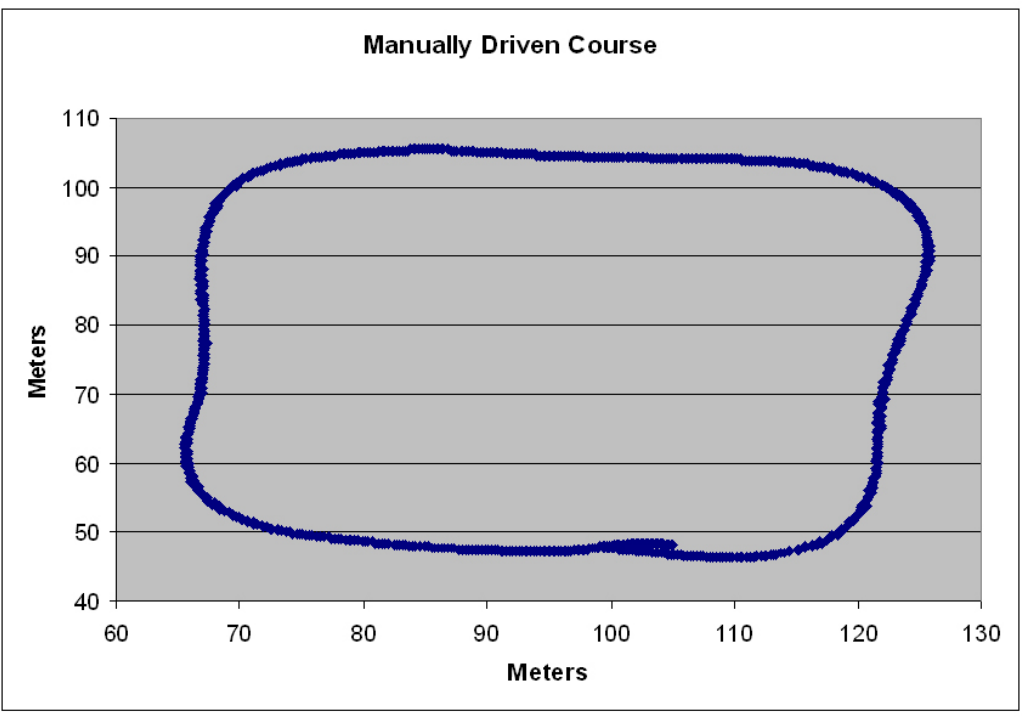

(b)

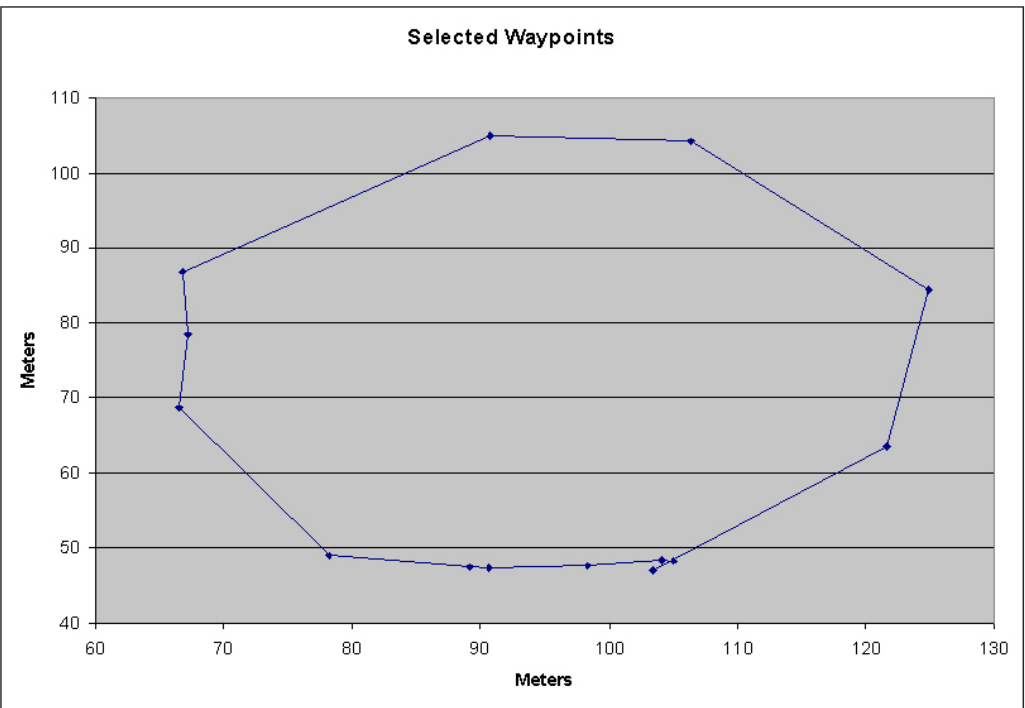

Figure 5.7: (a) A manually driven course with nearly 1000 waypoints. (b) The waypoints remaining after running the Point Reduction Algorithm on the manually driven course with a DP tolerance of 0.4 meters. These remaining waypoints are passed to the motion planner to create a list of trajectories to recreate the course.

trajectory incorrectly or at least undesirably or the PRA poorly selected a waypoint. To determine which was the case more testing was required.

The additional testing showed that the DP tolerance and the near-zero curvature tolerance have an impact on the waypoints selected by the PRA. The DP tolerance and near-zero curvature tolerance in Fig. 5.8a are 0.4 meters and $0.001 \frac{1}{m}$ 
(a)
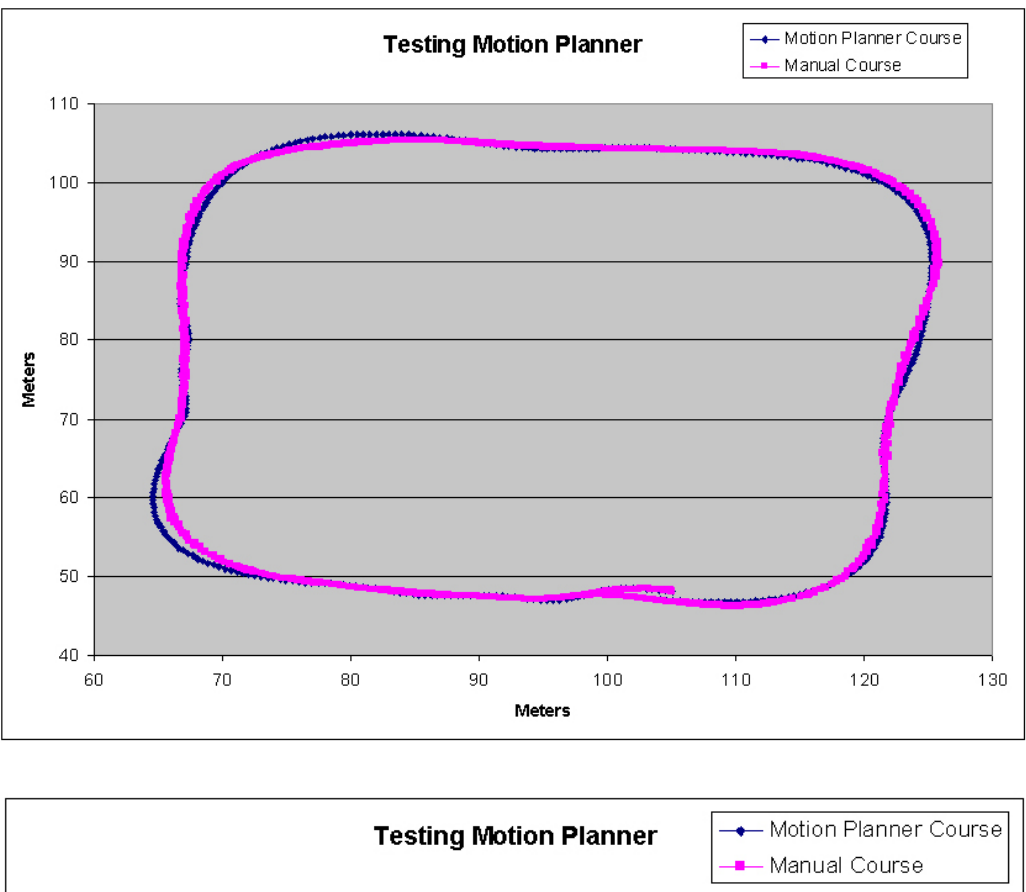

(b)

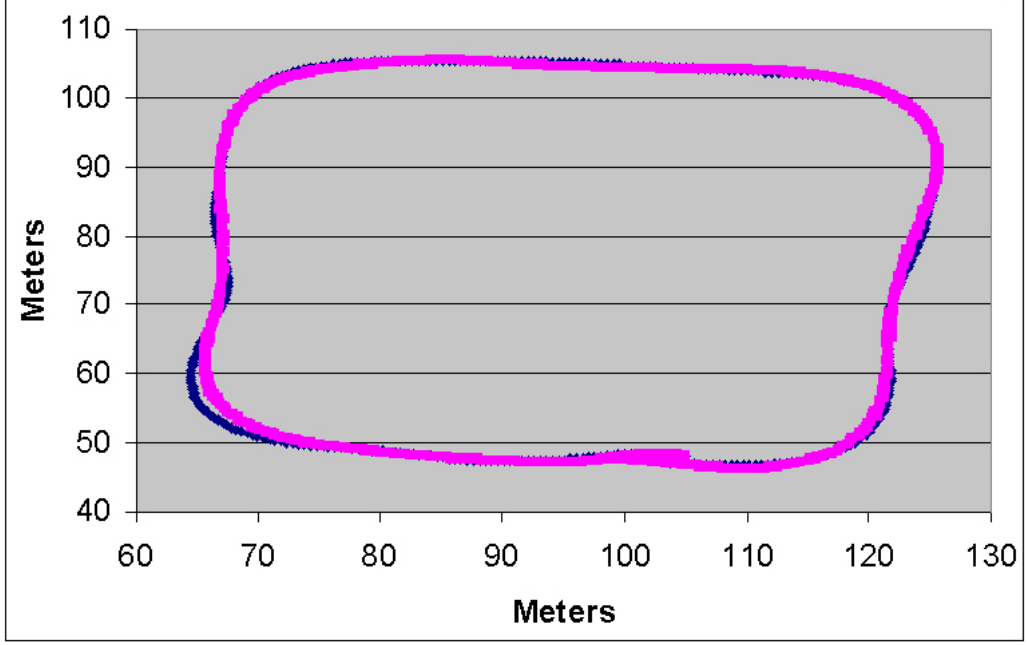

Figure 5.8: (a) Comparison of the manual driven course and the course created by the motion planner with a DP tolerance of 0.4 meters and a near-zero curvature tolerance of $0.001 \frac{1}{m}$. (b) Comparison of the manual driven course and the course created by the motion planner with a DP tolerance of 0.6 meters and a near-zero curvature tolerance of $0.01 \frac{1}{m}$.

respectively. Figure $5.8 \mathrm{~b}$ shows the same control data with a DP tolerance of 0.6 meters and a near-zero curvature tolerance of $0.01 \frac{1}{\mathrm{~m}}$. Here the results of the motion planner are better than that of the previous tolerances. (It should be noted that while $0.001 \frac{1}{m}$ may not seem very small, the radius of a circle with the same curvature would be 1000 meters.) While a curvature tolerance of $0.01 \frac{1}{m}$ may not be very reasonable, it 
does show that the PRA is providing the motion planner with a few poorly selected waypoints.

One possible source of the poorly selected waypoints could be that when a violation occurs within the motion planner the PRA picks the next waypoint in the list of zero or near-zero curvature waypoints. It is possible, and in the case of the lower left-hand corner of Fig. 5.8b most likely, that a better waypoint would be the previous waypoint and not the next waypoint in the list.

Another possibility of the poorly selected waypoints is that the recorded control data was poor or incorrect. Perhaps the GPS unit was not accurate enough due to a loss of one or more satellites or the compass was lagging on turns. Both cases would result in incorrect curvature calculations and therefore the PRA would be selecting waypoints based on bad data.

While not much can be done with bad data other than to delete it and record new good data, the PRA could be modified to pick new end waypoints both before and after the bad end waypoint. For example, if the motion planner returns the error that the waypoints are too close to generate a trajectory then the next waypoint in the list would be selected, However, if the motion planner returns the error that the

end waypoint heading cannot be achieved then perhaps the previous waypoint should be selected. A modified PRA is not presented here but is a matter of future work.

In the end the motion planner did what it was supposed to do, plan a trajectory between two waypoints that is smooth, natural, and drivable. While the some of the trajectories were not exactly desirable, it was a result of the PRA poorly choosing some waypoints and not the motion planner generating poor trajectories.

\subsection{Conclusion}

The Point Reduction Algorithm is used as a path planner to provide a few selected points and headings to the motion planner from a log file from either raw sensor data or the filtered state that was recored while manually driving the vehicle. The algorithm reads in the waypoints from the log file and removes all points with non-near-zero curvature. The Douglas-Peucker algorithm then selects all waypoints 
that exceed a given tolerance, and the remaining waypoints are passed to the motion planner to generate a list of trajectories that reproduce the test pattern. The trajectories can also be used by the controller to drive the vehicle and to test the vehicle system. Finally, some example tests of the motion planner show that, given good selected waypoints, it can accurately reproduce a manually driven course. 


\section{Chapter 6}

\section{Conclusion}

This thesis showed that the deflection $\delta$ of a clothoid determines its shape, and the curvature, $\kappa$ determines its size. The $x$ and $y$ components of the clothoid curve, and hence the length of the curve, all scale linearly with $\frac{1}{|\kappa|}$ while the proportions of the clothoid curve and its deflection stay the same. Thus, given the deflection of a curve, a clothoid curve can be created, and then given the length of its associated polyline, the curve can be scaled to the correct size without changing its deflection. This principle has been used to design the Clothoid_Turn() primitive algorithm that allows us to generate clothoids using constructive polylines. Using this method, paths are computed between an initial point and final point using only the coordinates of those points and their associated headings. This is the basis of the motion planner.

Building upon the Clothoid_Turn() algorithm, circular arcs were included to limit the upper bound of the curvature of a clothoid turn. The curvature of the circular arc is the maximum curvature that the vehicle can physically turn. And the angle of the circular arc is found using Newton's root approximation and iterating until a root is found.

Using constructive polylines and the Clothoid_Turn() algorithm with circular arcs, a clothoid set has been developed consisting of two clothoid curves which is a turn similar to a "C" path by Reeds and Shepp [8], except that it uses clothoid arcs as well as circular arcs. The u-turn is a special case of this type of path.

A generic turn algorithm was also developed that produces a path consisting of two clothoid sets similar to a "CC" path by Reeds and Shepp [8]. It has also been shown that the straight-curve turn, the curve-straight turn, and the lane change are 
all special cases of the generic turn. All of the maneuvers discussed in Section 4.3 are smooth, natural, and drivable trajectories.

Finally, it was shown how a log file containing waypoints of a test course can be used to test the motion planner and vehicle system. The Point Reduction Algorithm selects a few waypoints from a log file and passes the selected waypoints to the motion planner. The motion planner is then tested to see if it can reproduce the test pattern. The vehicle system and controls can also be tested by driving the trajectories generated by the motion planner.

\subsection{Accomplishments}

The major accomplishments of this thesis are the use of construction polylines to scale clothoids while retaining a clothoid's proportions. Smooth, natural, drivable trajectories are generated by using a combination of lines, circular arcs, and scaled clothoids. The motion planner also determines what type of maneuver to execute by comparing the trajectory deflection and the angle between the initial heading and the construction polyline. And finally, the development of the Point Reduction Algorithm to test the motion planner and vehicle system by selecting a few waypoints from a file containing waypoints and passing the points to the motion planner, which can (if working properly) recreate the course that the waypoints in the file describe in both software and hardware.

\subsection{Future Research}

Future research includes developing paths using the Clothoid_Turn() algorithm that include more than two polylines like those path sets described by Reeds and

Shepp [8] and allowing for reversal in direction using free parameter $q$, and characterizing feasible paths by finding a function that will return the minimal length of $d$ required for a valid trajectory (Eq. 4.28). Future work also includes the continued development of a set of heuristics to determine the type of maneuver to perform, how to parameterize the generic turn for different maneuvers, and to modify the Point 
Reduction Algorithm to choose either previous or next waypoints from the list of zero and near-zero curvatures. 


\section{Bibliography}

[1] I. Kolmanovsky and N. McClamroch, "Development in nonholonomic control problems," IEEE Control Systems, vol. 15, no. 6, pp. 20-36, 1995. 1

[2] D. Coombs, K. Murphy, A. Lacaze, and S. Legowik, "Driving autonomously offroad up to $35 \mathrm{~km} / \mathrm{h}$," in Proceedings of the IEEE Intelligent Vehicles Symposium. IEEE, October 2000, pp. 186-191. 1

[3] D. Knowles, "Real time continuous curvature path planner for an autonomous vehicle in an urban environment," California Institute of Technology, Tech. Rep., September 2006. 1

[4] M. Pivtoraiko and A. Kelly, "A study of polynomial curvature clothoid paths for motion planning for car-like robots," The Robotics Institute Carnegie Mellon University, 5000 Forbes Avenue, Pittsburgh, PA 15213, Tech. Rep. CMU-RI-TR05-nn, December 2004. 1

[5] D. H. Shin and S. Singh, "Path generation for robot vehicles using composite clothoid segments," Robotics Institute, Carnegie Mellon University, Pittsburgh, PA, Tech. Rep. CMU-RI-TR-90-31, December 1990. 1, 2, 3

[6] T. Fraichard and A. Scheuer, "From Reeds and Shepp's to continuous-curvature paths," Robotics, IEEE Transactions on, vol. 20, no. 6, pp. 1025-1035, Dec. 2004. 1, 2, 3, 11, 18, 44

[7] L. Dubins, "On curves of minimal length with a constraint on average curvature, and with prescribed initial and terminal positions and tangents," American Journal of Mathematics, vol. 79, pp. 497-517, 1957. 2, 4

[8] J. Reeds and L. Shepp, "Optimal paths for a car that goes both forwards and backwards," Pacific Journal of Mathematics, vol. 145, no. 2, pp. 367-393, 1990. $2,4,41,65,66$

[9] J. H. Henrie and D. K. Wilde, "Planning continuous curvature paths using constructive polylines," Journal of Aerospace Computing, Information, and Communication, vol. 4, no. 12, pp. 1143-1157, 2007. 5

[10] D. Walton and D. Meek, "A controlled clothoid spline," Computers and Graphics, vol. 29, no. 3, pp. 353-363, 2005.

7,22 
[11] B. B. Kimia, I. Frankel, and A.-M. Popescu, "Euler spiral for shape completion," International Journal of Computer Vision, vol. 54, no. 1-3, pp. 157-180, 2003. 9

[12] DARPA, "Urban Challenge Rules," Defense Advanced Research Projects Agency, pp. 2-8, October 2007. [Online]. Available: http://www.darpa.mil/ grandchallenge/rules.asp 38, 40

[13] D. Douglas and T. Peucker, "Algorithms for the reduction of the number of points required to represent a digitized line or its caricature," The Canadian Cartographer, vol. 10, no. 2, pp. 112-122, 1973. 54

[14] N. Schuurman, GIS: A Short Introduction. Wiley-Blackwell, April 2004. 56

[15] D. Sunday, "Polyline simplification," Internet, April 2007. [Online]. Available: http://www.softsurfer.com/algorithms.htm 56, 71

[16] E. M. Joao, Causes and Consequences of Map Generalisation. Boca Raton, FL: CRC Press, 1998. 56

[17] D. Sunday, "C++ point and vector class," Internet, April 2007. [Online]. Available: http://www.softsurfer.com/algorithms.htm 71

[18] J. N. McElwaine, "Calculate Fresnel sin and cos integrals," Internet, January 2007. [Online]. Available: http://www.damtp.cam.ac.uk/user/jnm11/software/ fresnel.m 71 


\section{Appendix}

\section{File Listing}

The following is a list and description of the files uploaded with electronic edition of this thesis.

\section{Header Files File Description}

CIN_recv.h Structure definitions used by Global_Memory.cpp.

common.h Common definitions used by the Douglas-Peucker algorithm [17].

Drivepoint.h Structure definitions and function declarations used by the motion planner.

point.h Point Class definitions used by the Douglas-Peucker algorithm [17].

vector.h Vector Class definitions used by the Douglas-Peucker algorithm [17].

\section{Program Files}

Fresnel2.cpp

Global_Memory.cpp

GPSsimplify.cpp

Motion_Planner.cpp

peucker.cpp

point.cpp

vector.cpp

\section{File Description}

Fresnel sine and cosine integrals [18].

Contains functions for creating trajectory and drive point memory structures.

Contains the Point Reduction Algorithm.

Contains the motion planner algorithm, Clothoid_Turn(), and Generic_Turn().

Implementation of the Douglas-Peucker algorithm [15].

Point Class API used by the Douglas-Peucker algorithm [17].

Vector Class API used by the Douglas-Peucker algorithm [17]. 